\title{
Sensitivity Enhancement by Multiple-Contact Cross-Polarization under Magic-Angle Spinning
}

J. Raya and J. Hirschinger*

Institut de Chimie, UMR 7177 CNRS, Université de Strasbourg, Strasbourg, France

Submitted to the Journal of Magnetic Resonance

Revised manuscript

*Corresponding author

E-mail address : $\underline{\text { hirschinger@unistra.fr }}$ 


\begin{abstract}
Multiple-contact cross-polarization (MC-CP) is applied to a powder samples of ferrocene and L-alanine under magic-angle spinning (MAS) conditions. The method is described analytically through the density matrix formalism. The combination of a two-step memory function approach and the Anderson-Weiss approximation is found to be particularly useful to derive approximate analytical solutions for single-contact Hartmann-Hahn CP (HHCP) and MC-CP dynamics under MAS. We show that the MC-CP sequence requiring no pulse-shape optimization yields higher polarizations at short contact times than optimized adiabatic passage through the $\mathrm{HH}$ condition $\mathrm{CP}$ (APHH-CP) when the MAS frequency is comparable to the heteronuclear dipolar coupling, i.e., when APHH-CP through a single sideband matching condition is impossible or difficult to perform. It is also shown that the MC-CP sideband $\mathrm{HH}$ conditions are generally much broader than for single-contact HHCP and that efficient polarization transfer at the centerband $\mathrm{HH}$ condition can be reintroduced by rotor-asynchronous multiple equilibrations-re-equilibrations with the proton spin bath. Boundary conditions for the successful use of the MC-CP experiment when relying on spin-lattice relaxation for repolarization are also examined.
\end{abstract}

Keywords : Solid-state NMR ; Cross-polarization ; Magic-angle spinning ; Spin diffusion 


\section{Introduction}

Since the pioneering work by Pines, Gibby and Waugh [1;2], crosspolarization $(\mathrm{CP})$ has been the most widely used solid-state NMR technique for enhancing the nuclear magnetization of rare spins $S$ with a low gyromagnetic ratio such as ${ }^{13} \mathrm{C}$ and ${ }^{15} \mathrm{~N}$ from abundant spins $I$ with a higher gyromagnetic ratio $\left(\right.$ e.g., $\left.{ }^{1} \mathrm{H}\right)$ under static and magic-angle spinning (MAS) conditions $[3 ; 4 ; 5 ; 6]$. However, it is well known that the $\mathrm{CP}$ transfer efficiency is strongly reduced when the MAS rate exceeds both the homonuclear $(I-I)$ and heteronuclear $(I-S)$ dipolar couplings. Indeed, at these spinning speeds, the normally broad Hartmann-Hahn $(\mathrm{HH})$ condition [7] splits into a series of new « sideband » matching conditions spaced at the MAS frequency [8]. With increasing spinning rate, polarization transfer becomes ineffective at centerband $\mathrm{HH}$ matching and efficient $\mathrm{CP}$ is obtained only at the firstand second-order sidebands $[9 ; 10 ; 11]$. In practice, sideband $\mathrm{HH}$ matching is difficult because of the excessive sensitivity of the matching conditions to the radiofrequency (RF) field amplitudes of the two channels. To overcome these problems, several techniques such as simultaneous phase-inversion CP (SPICP) [12], amplitude-modulated CP (AMCP) [13], synchronous accumulation of polarization (SAP) [14], variable-amplitude CP (VACP) [15], ramped-amplitude CP (RAMP-CP) [16], variable-effective-field CP (VEFCP) [17], frequency-sweep CP (FSCP) [18], adiabatic passage through the HH condition (APHH) [19], amplitude-modulated adiabatic-passage CP (AMAP-CP) [20], frequency-modulated CP (FMCP) [21], nuclear integrated CP (NICP) [22], simultaneous adiabatic spin-locking CP (SADISCP) [23], rotor echo short pulse irradiation mediated CP $\left({ }^{\text {RESPIRATION }} \mathrm{CP}\right)$ [24], and adiabatic ${ }^{\text {RESPIRATION }} \mathrm{CP}$ [25] have been developed. An important issue in the application of any analytical technique is the quantitative reliability of the data. In CP experiments, the $S$-spin magnetization comes from the $I$-spin reservoir. Thus, it has long been recognized that the signal intensities may disagree with the atomic ratios 
for many reasons [26]. One of the major problems arises from the competition between the $\mathrm{CP}$ and spin-lattice relaxation in the rotating frame. Different chemical groups generally exhibit different $\mathrm{CP}$ build-up rates and spin-lattice relaxation times in the rotating frame so that line intensity ratios depend critically on the $\mathrm{CP}$ contact time. Hence, in principle, reliable relative peak intensities within a CP spectrum cannot be compared without a careful study of their CP dynamics by variable contact time experiments [6]. Methods for obtaining « quantitative » CPMAS spectra have nevertheless been proposed over the past decades $[27 ; 28 ; 29 ; 30 ; 31 ; 32 ; 33 ; 34]$. Among these techniques, the so-called multiple-contact CP (MC-CP) scheme originally proposed in the textbook by Gerstein and Dybowski [35] has been recently shown to provide quantitative ${ }^{13} \mathrm{C}$ NMR spectra of complex organic materials [34] as well as an enhancement of the $I-S$ magnetization transfer when compared to HHCP and APHH-CP in ${ }^{15} \mathrm{~N}$ NMR of membrane proteins reconstituted in magnetically aligned bicelles [36; 37] and ${ }^{13} \mathrm{C}$ NMR under slow MAS [38].

In this paper, we apply MC-CP under MAS to powder samples of ferrocene and $L$-alanine and compare the results with the ones obtained with HHCP and APHH-CP. In these systems, the heteronuclear ${ }^{1} \mathrm{H}-{ }^{13} \mathrm{C}$ coupling between directly bonded atoms exceeds all other couplings. Both the reintroduction of efficient $\mathrm{CP}$ at the centerband $\mathrm{HH}$ condition and the broadening of the $\mathrm{HH}$ conditions are examined. The experimental results are directly compared with analytical and numerical calculations obtained by using the so-called $I-I^{*}-S$ model $[6 ; 39 ; 40 ; 41]$ for CP dynamics based on a coherent description of the dipolar-coupled $I^{*}-S\left({ }^{1} \mathrm{H}^{13} \mathrm{C}\right)$ spin pair and an incoherent description of the interaction of the $I^{*}$ spin with a large number of other $I$ spins.

\section{Theory}


This section is organized into three subsections as follows. Subsection 2.1. gives a brief description of the $I-I *_{-} S$ model. Analytical solutions to CP dynamics are then derived using the fast fluctuation (or Markoff) approximation (subsection 2.2.). and considering memory (or non-Markoffian) effects of the $I$-spin bath (subsection 2.3.).

\subsection{The I-I*-S model}

In order to describe the spin dynamics, we use the $I-I^{*}-S$ model originally proposed by Müller, Kumar, Baumann and Ernst (MKBE) [40]. The system is then treated as a tightly coupled $I^{*} S\left(e . g .,{ }^{1} \mathrm{H}_{-}{ }^{13} \mathrm{C}\right)$ spin pair immersed in a spin-bath consisting of the remaining $I$ spins. The model assumes that only one spin $I^{*}$ interacts with the $I$-spin bath or infinite energy reservoir of $I$ spins which is described in a phenomenological way. The modeled Hamiltonian in the tilted double rotating frame is

$$
\hat{H}=\hat{H}_{S}+\hat{H}_{S E}+\hat{H}_{E} .
$$

The system (S) Hamiltonian of the coupled $I^{*}$ and $S$ spins is

$$
\hat{H}_{S}=\omega_{1 I} \hat{I}_{z}+\omega_{1 S} \hat{S}_{z}+2 b \hat{I}_{x} \hat{S}_{x},
$$

where $\omega_{1 I}$ and $\omega_{1 S}$ are the amplitudes of the radiofrequency (RF) fields applied to the $I$ and $S$ spins. The system (heteronuclear) dipolar coupling between the $I^{*}$ and $S$ spins is given by

$$
b=-\frac{\gamma_{I} \gamma_{S} \hbar}{2 r^{3}}\left(3 \cos ^{2} \theta-1\right),
$$

where $r$ is the $I^{*}-S$ internuclear distance and $\theta$ is the angle between the internuclear vector $\boldsymbol{r}$ and the static magnetic field $\boldsymbol{B}_{\mathbf{0}}$. Note that, for the $\mathrm{C}_{5} \mathrm{H}_{5}$ rings of ferrocene undergoing fast uniaxial rotational motion around the five-fold molecular symmetry axis, the dipolar interactions are partially averaged so that $b$ must be scaled by the order parameter of the motion $(-1 / 2)$ and $\theta$ is converted into the angle between the 
axis of free molecular rotation and $\boldsymbol{B}_{\mathbf{0}}$. In the expression of $\hat{H}_{S}$, the resonance offsets are neglected.

$\hat{H}_{S E}$ is the $I-I^{*}$ system-environment (SE) interaction Hamiltonian

$$
\hat{H}_{S E}=\alpha \hat{I}_{z} \hat{F}_{z}+\frac{1}{2} \beta\left(\hat{I}_{+} \hat{F}_{-}+\hat{I}_{-} \hat{F}_{+}\right)=\alpha \hat{I}_{z} \hat{F}_{z}+\beta\left(\hat{I}_{x} \hat{F}_{x}+\hat{I}_{y} \hat{F}_{y}\right)
$$

where the bath operators are defined by

$$
\hat{F}_{ \pm}=\hat{F}_{x} \pm i \hat{F}_{y} \quad \text { and } \quad \hat{F}_{u}=\sum_{k} d_{k} \hat{I}_{k u}, \quad \text { with } \quad u=x, y, z
$$

$\hat{H}_{S E}$ is an Ising interaction if $\beta=0$ and an XY, isotropic (Heisenberg) or the truncated dipolar interaction if $\alpha / \beta=0,1,-2$, respectively. This last case is typical in solid-state NMR experiments $[42 ; 43 ; 44 ; 45]$.

$\hat{H}_{E}$ is the $I$-spin bath or environment (E) Hamiltonian

$$
\hat{H}_{E}=\omega_{1 I} \sum_{k} \hat{I}_{k z}+\sum_{k>j} d_{j k}\left[2 \hat{I}_{j z} \hat{I}_{k z}-\frac{1}{2}\left(\hat{I}_{j+} \hat{I}_{k-}+\hat{I}_{j-} \hat{I}_{k+}\right)\right] .
$$

The SE homonuclear dipolar coupling constants $d_{k}$ between the $I^{*}$ spin and the spins $I_{k}$ are given by

$$
d_{k}=-\frac{\gamma_{I}^{2} \hbar}{2 r_{k}^{3}}\left(3 \cos ^{2} \theta_{k}-1\right)
$$

The (homonuclear) dipolar couplings $d_{j k}$ between the $I$ spins of the bath are

$$
d_{j k}=-\frac{\gamma_{I}^{2} \hbar}{2 r_{j k}^{3}}\left(3 \cos ^{2} \theta_{j k}-1\right) .
$$

$r_{k}$ and $r_{j k}$ are internuclear distances and $\theta_{k}$ and $\theta_{j k}$ are angles between internuclear vectors and $\boldsymbol{B}_{\mathbf{0}}$. In case of strong irradiation on the $I$ spins $\left(\omega_{1 I}>>\left|d_{j k}\right|\right)$, non-secular terms with respect to the large RF field Hamiltonian may be ignored so that $\hat{H}_{E}$ is usually rewritten as 


$$
\hat{H}_{E}=\omega_{1 I} \sum_{k} \hat{I}_{k z}-\frac{1}{2} \sum_{k>j} d_{j k}\left[2 \hat{I}_{j z} \hat{I}_{k z}-\frac{1}{2}\left(\hat{I}_{j+} \hat{I}_{k-}+\hat{I}_{j-} \hat{I}_{k+}\right)\right]
$$

While the terms $\hat{F}_{u}$ are bath operators in a quantum mechanical relaxation theory they represent classical stochastic forces in the semi-classical theory [46; 47; 48; 49]. The experimental conditions justify a high temperature approximation, and hence the semi-classical theory coincides with a quantum treatment $[46 ; 47 ; 48 ; 49]$. Since $\hat{H}_{E}$ does not commute with $\hat{H}_{S E}$ the system experiences a coupling to the environment that fluctuates by virtue of flip-flop transitions within the $I$-spin bath produced by terms of the type $I_{j \pm} I_{k \mp}$ in Eqs (6) or (9). For a semi-classical treatment, we trace over the bath variables and replace the bath operators $\hat{F}_{u}$ by the stochastic functions $F_{u}(t)$ representing classical random local fields with a Gaussian distribution. The random SE interaction Hamiltonian is then written as

$$
\hat{H}_{S E}(t)=\alpha F_{z}(t) \hat{I}_{z}+\beta\left[F_{x}(t) \hat{I}_{x}+F_{y}(t) \hat{I}_{y}\right]
$$

The time average of these random processes satisfies [46]

$$
\overline{F_{u}(t)}=\operatorname{Tr}_{E}\left\{\hat{F}_{u}(t) \hat{\rho}_{E}(0)\right\}=0,
$$

and their stationary time correlation functions which depend only on the time difference $t-t^{\prime}=\tau$ are [46]

$$
K_{u v}(\tau)=\operatorname{Tr}_{E}\left\{\hat{F}_{u}(t) \hat{F}_{v}\left(t^{\prime}\right) \hat{\rho}_{E}(0)\right\}=\overline{F_{u}(\tau) F_{v}(0)},
$$

with $u, v=x, y, z$.

The bar above a quantity is a shorthand notation for the ensemble average, $\operatorname{Tr}_{E}$ denotes a partial trace over the environment variables and $\hat{\rho}_{E}(0)$ is the density matrix of the spin-bath assumed to be a state of thermal equilibrium [46; 49]. Assuming that the spatial directions are statistically independent, we have $K_{u v}(\tau)=0$ if $u \neq v$. Moreover, considering that all the bath spins are equivalent, the decay of $K_{u u}(\tau)$ is controlled by the same bath correlation time $\tau_{B}$. In our system, $\tau_{B}$ depends on the strength of the dipolar couplings $d_{j k}$ between the ${ }^{1} \mathrm{H}$ nuclear spins of the bath ( $c f$. Eq. 
(6)). Since $\hat{H}_{E}$ is scaled by the term $P_{2}\left(\cos \left(90^{\circ}\right)\right)=-1 / 2$ in the presence of a strong applied RF field $\omega_{1 I}$ (cf. Eq.(9)) note that the correlation time of the $I$-spin bath in the rotating frame $\tau_{x}$ is, in principle, larger than $\tau_{B}$ by a factor of 2 [50].

\subsection{Fast fluctuation (or Markoff) approximation}

\subsubsection{Exact-powder secular solutions}

Following the usual treatment of second-order perturbation theory and neglecting $T_{1 \rho}$ relaxation, the dynamics of the reduced density operator is given by the generalized Liouville - von Neumann differential equation $[39 ; 40 ; 41 ; 47 ; 48]$

$$
\frac{d}{d t} \hat{\sigma}(t)=-i\left[\hat{H}_{S}, \hat{\sigma}(t)\right]-\hat{\hat{\Gamma}}\{\hat{\sigma}(t)-\hat{\sigma}(\infty)\}
$$

where

$$
\hat{\sigma}(t)=\operatorname{Tr}_{E}\{\hat{\rho}(t)\}
$$

is the reduced density operator, $\hat{\rho}(t)$ representing the density operator of the total spin system. The initial and final density operators, $\hat{\sigma}(0)$ and $\hat{\sigma}(\infty)$, are $\hat{I}_{z}$ and $\hat{I}_{z}+$ $\hat{S}_{z}$, respectively. The spin diffusion (SD) superoperator in the rotating frame $\hat{\hat{\Gamma}}$, which is a relaxation superoperator [48], is given by the $I-I^{*} \mathrm{SE}$ interaction. It accounts for the dissipative interactions between the reduced spin system and the spin-bath and it imposes the relaxation of the density operator towards its equilibrium value $\hat{\sigma}(\infty)$. It is further assumed that the correlation time of the spin bath $\tau_{x}$ is short (Markoff approximation) [46], i.e., $d_{S E} \tau_{x} \ll 1$ where the SE homonuclear coupling strength is defined by

$$
d_{S E}=\sqrt{\sum_{k} d_{k}^{2}} .
$$

In this extreme narrowing regime or fast fluctuation (FF) approximation, the spin diffusion superoperator $\hat{\hat{\Gamma}}$ can be written as $[39 ; 40 ; 41]$ 


$$
\hat{\hat{\Gamma}}(\hat{\sigma})=R_{d p}\left[\hat{I}_{z},\left[\hat{I}_{z}, \hat{\sigma}\right]\right]+R_{d f}\left\{\left[\hat{I}_{x},\left[\hat{I}_{x}, \hat{\sigma}\right]\right]+\left[\hat{I}_{y},\left[\hat{I}_{y}, \hat{\sigma}\right]\right]\right\}
$$

The elements of the SD supermatrix then depend on two rate constants, $R_{d p}=1 / T_{d p}$ and $R_{d f}=1 / T_{d f}$, that are, in principle, orientation dependent. As $R_{d f}$ is associated with the flip-flop term of the homonuclear $\left(I-I^{*}\right)$ dipolar Hamiltonian (XY or planar term) [44] its role in transferring polarization can be easily interpreted. Hence, $R_{d f}$ allows the complete thermal equilibration with the bath (« effective SD process »$d f)$. The effect of $R_{d p}$ is more subtle : it can be associated with a process where the environment observes the system breaking its coherences (Ising term) [43] driving the system to the internal quasiequilibrium [51]. Therefore, $R_{d p}$ only acts on the decay or damping ( $d p)$ of the coherences. Note that $R_{d p}$ and $R_{d f}$ contain the different sources of anisotropy of the $\mathrm{SE}$ interaction. The usual approximation considers $R_{d p}=$ $R_{d f}$ (isotropic $I-I^{*} \mathrm{SE}$ interaction). A better approximation considers a dipolar I-I* SE interaction Hamiltonian, i.e., $T_{d f} / T_{d p}=R_{d p} / R_{d f}=\alpha^{2} / \beta^{2}=4[42 ; 43 ; 44 ; 45]$.

When the amplitudes of the applied RF fields are much larger than the $I^{*}-S$ dipolar coupling $\left(\omega_{1 I}+\omega_{1 S}>|b|\right)$, the non-secular elements of the dipolar interaction with respect to $I_{z}+S_{z}$ can be neglected. The system Hamiltonian then becomes

$$
\begin{aligned}
\hat{H}_{S}= & \frac{\Sigma}{2}\left(\hat{I}_{z}+\hat{S}_{z}\right)+\frac{\Delta}{2}\left(\hat{I}_{z}-\hat{S}_{z}\right)+\frac{b}{2}\left(\hat{I}_{+} \hat{S}_{-}+\hat{I}_{-} \hat{S}_{+}\right) \\
& =\Sigma \hat{I}_{z}^{\Sigma}+\Delta \hat{I}_{z}^{\Delta}+b \hat{I}_{x}^{\Delta},
\end{aligned}
$$

where $\Sigma=\omega_{1 I}+\omega_{1 S}$ and $\Delta=\omega_{1 I}-\omega_{1 S}$.

The operators $\hat{I}_{u}^{\Sigma}$ and $\hat{I}_{u}^{\Delta}(u=x, y, z)$ are fictitious spin- $1 / 2$ operators acting in the double quantum (DQ) and the zero quantum (ZQ) subspace, respectively [51; 52]. Now, it is evident that the dipolar interaction is an XY (flip-flop) term that splits the energy level of the states $|+-\rangle$ and $|-+\rangle$ and induces an oscillation between them. 
The DQ operator $\Sigma \hat{I}_{z}^{\Sigma}$ commutes with the ZQ part of $\hat{H}_{S}$ and the density operator at initial time $\hat{\sigma}(0)=\hat{I}_{z}$. As a consequence, the $\Sigma \hat{I}_{z}^{\Sigma}$ term can be eliminated and only the secular part of the $I^{*}-S$ spin-pair Hamiltonian, $\hat{H}_{I S}^{(0)}=b \hat{I}_{x}^{\Delta}$, operates at the $\mathrm{HH}$ condition $(\Delta=0)$ in strong RF fields. In addition, $\hat{H}_{I S}^{(0)}$ commutes with the final density operator $\hat{\sigma}(\infty)=\hat{I}_{z}+\hat{S}_{z}$. The QM master equation (Eq. (14)) can then be written in the following homogeneous form

$$
\left.\left.\left.\frac{d}{d t}|\hat{\sigma}(t)\rangle\right\rangle=-i \hat{\hat{L}}|\Delta \hat{\sigma}(t)\rangle\right\rangle=-\left[i \hat{\hat{H}}_{I S}^{(0)}+\hat{\hat{\Gamma}}\right]|\Delta \hat{\sigma}(t)\rangle\right\rangle
$$

where $|\hat{\sigma}(t)\rangle\rangle$ is the reduced density operator in Liouville space and $|\Delta \hat{\sigma}(t)\rangle\rangle=$ $|\hat{\sigma}(t)\rangle\rangle-|\hat{\sigma}(\infty)\rangle\rangle$.

A general non-secular (NS) analytical solution to Eq. (18) for the $S$-spin polarization $\left\langle S_{z}\right\rangle(t)$ has been obtained by Alvarez et al. [43] for arbitrary values of $R_{d p}$ and $R_{d f}$ in the case of a static sample.

The heteronuclear dipolar coupling constant between the $I^{*}$ and $S$ spins is time dependent in the presence MAS :

$$
b(t)=\frac{D_{I S}}{2}\left\{\sqrt{2} \sin 2 \beta \cos \left(\gamma+\omega_{r} t\right)-\sin ^{2} \beta \cos \left(2 \gamma+2 \omega_{r} t\right)\right\},
$$

where $D_{I S}=\gamma_{I} \gamma_{S} \hbar / r^{3}, \beta$ is the angle between $\boldsymbol{r}$ and the spinning axis, $\gamma$ is an initial azimuth of $\boldsymbol{r}$ about that axis and $\omega_{r} / 2 \pi=v_{r}$ is the spinning frequency. Assuming that the Markoff or FF approximation $\left(d_{S E} \tau_{x}<1\right)$ is still valid, the QM master equation (Eq. (18)) is extended to the MAS case simply replacing $b$ by $b(t)$. Although the time dependence of $b(t)$ makes the derivation of NS solutions to the QM master equation a formidable task $[53 ; 54]$ a simple solution is readily obtained in the secular approximation, i.e., when the $I^{*}-S$ dipolar coupling constant is much higher than the SD rate constants ( $D_{I S} \gg R_{d p}$ and $R_{d f}$ ). Indeed, the system superoperator $\hat{\hat{L}}(t)$ evaluated at different times then commutes with itself $\left(\left[\hat{\hat{L}}\left(t^{\prime}\right), \hat{\hat{L}}\left(t^{\prime \prime}\right)\right]=0\right)[55]$.

Eq. (18) then yields the following formal solution 


$$
\begin{aligned}
&|\Delta \hat{\sigma}(t)\rangle\rangle\left.\left.=\exp \left[-i \int_{0}^{t} \hat{\hat{L}}\left(t^{\prime}\right) d t^{\prime}\right] \Delta \hat{\sigma}(0)\right\rangle\right\rangle \\
&\left.\left.=\exp \left[-i \int_{0}^{t} \hat{\hat{H}}_{I S}^{(0)}\left(t^{\prime}\right) d t^{\prime}-\hat{\hat{\Gamma}}^{(0)} t\right] \Delta \hat{\sigma}(0)\right\rangle\right\rangle,
\end{aligned}
$$

where $\hat{\hat{\Gamma}}^{(0)}$ is the SD superoperator in the rotating frame obtained after neglecting the rapidly oscillating NS terms with respect to $\hat{\hat{H}}_{I S}^{(0)}[55]$.

The $S$ spin polarization as a function of the contact time is then easily calculated

$$
\left\langle S_{z}\right\rangle(t)=\left\langle\left\langle\hat{\sigma}(t) \mid \hat{S}_{z}\right\rangle\right\rangle=1-\frac{1}{2} \exp \left(-R_{d f} t\right)-\frac{1}{2} \exp \left[-\left(R_{d f}+\frac{R_{d p}}{2}\right)\right] t \cos \left(\int_{0}^{t} b\left(t^{\prime}\right) d t^{\prime}\right),
$$

where

$$
\int_{0}^{t} b\left(t^{\prime}\right) d t^{\prime}=\frac{D_{I S}}{2}\left\{\frac{\sqrt{2} \sin 2 \beta}{\omega_{r}}\left[\sin \left(\gamma+\omega_{r} t\right)-\sin \gamma\right]-\frac{\sin ^{2} \beta}{2 \omega_{r}}\left[\sin \left(2 \gamma+2 \omega_{r} t\right)-\sin 2 \gamma\right]\right\} .
$$

This « MKBE-MAS » solution has been used previously by Tekely and co-workers $[56 ; 57 ; 58]$ to simulate cross-polarization/polarization inversion (CPPI) MAS spectra. Its range of validity under slow MAS conditions has been evaluated by comparison with exact numerical integration of the QM master equation in Ref. [55]. 


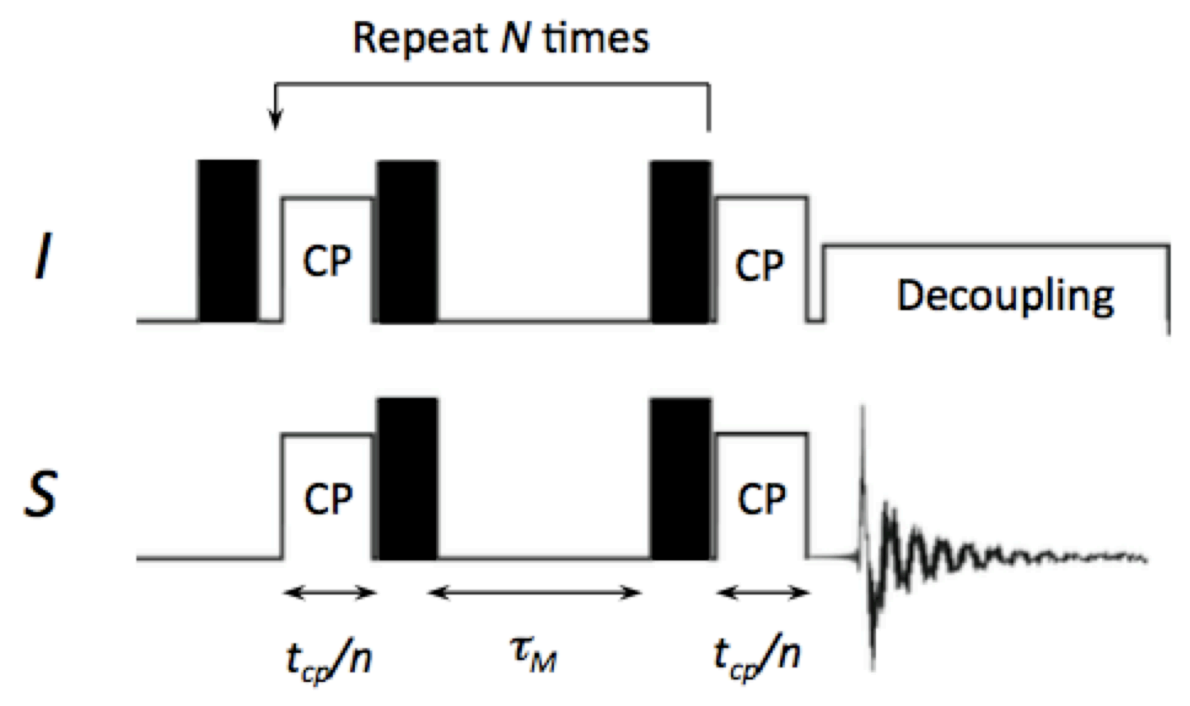

Figure 1 : Schematic representation of the pulse sequence for Multiple-Contact Cross-Polarization (MC-CP). The filled rectangles indicate $\pi / 2$ pulses.

The MC-CP experiment is shown in Fig. 1. After the initial CP step, both the $I$ and $S$ magnetizations are stored along the static magnetic field $\boldsymbol{B}_{\mathbf{0}}$ by «flip-back $\pi / 2$ pulses. During the mixing time $\tau_{M}$, the directly bound $I^{*}$ spin repolarizes through SD with the remaining $I$ spins while the $S$ magnetization is retained $\left(T_{1}\right.$ spin-lattice relaxation and SD among the $S$ spins is neglected during $\tau_{M}$ ). In other words, the local magnetization loss of the $I^{*}$ spin due to the rapid polarization transfer to the $S$ spin during CP is compensated solely by SD from the neighboring $I$ spins $\left(\tau_{M}<<T_{1}^{I}\right)$. This contrasts with previous uses of the MC-CP scheme relying on $T_{1}^{I}$ relaxation for equilibration-re-equilibration of the $I$ spins to the lattice temperature during each mixing step $\left(\tau_{M}>>T_{1}^{I}\right)[27 ; 34 ; 36]$. The $I$ and $S$ magnetizations are then returned to the transverse plane and the polarization transfer is repeated again. After $N$ cycles, i.e., $n=N+1 \mathrm{CP}$ contacts of duration $t_{c p} / n$, the free induction decay of the $S$ spins is observed. Eq. (20) then yields the following formal solution after $n \mathrm{CP}$ steps of the MC-CP sequence : 


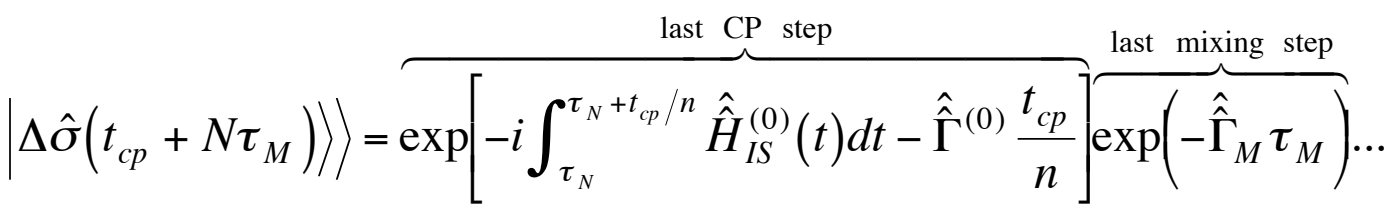

$$
\begin{aligned}
& \overbrace{\times \exp \left[-i \int_{\tau_{1}}^{\tau_{1}+t_{c p} / n} \hat{\hat{H}}_{I S}^{(0)}(t) d t-\hat{\hat{\Gamma}}^{(0)} \frac{t_{c p}}{n}\right]}^{\text {second } \mathrm{CP} \text { step }} \overbrace{\exp \left(-\hat{\hat{\Gamma}}_{M} \tau_{M}\right)}^{\text {first mixing step }} \\
& \overbrace{\times \exp \left[-i \int_{0}^{t_{c p} / n} \hat{\hat{H}}_{I S}^{(0)}(t) d t-\hat{\hat{\Gamma}}^{(0)} \frac{t_{c p}}{n}\right]}^{\text {first CP step }} \Delta \hat{\sigma}(0)\rangle\rangle
\end{aligned}
$$

where $\tau_{k}=k\left(\frac{t_{c p}}{n}+\tau_{M}\right)$.

$\hat{\hat{\Gamma}}_{M}$ is the SD superoperator in the laboratory frame operating during $\tau_{M}$ which has the same form as $\hat{\hat{\Gamma}}$ (Eq. (16)), the rate constants defining the corresponding SD supermatrix being nevertheless expected to be higher since spin diffusion in the laboratory frame is faster than in the rotating frame by a factor of $\sim 2$ to $\sim 4[39 ; 59$; $60]$. Since the $I^{*}-S$ dipolar Hamiltonian superoperator has no effect on the relevant coherences during $\tau_{M}$ it has been dropped from Eq. (23). Under the following assumptions, Eq. (23) gives simple expressions for the $S$-spin polarization at the end of the MC-CP sequence :

(i) No re-equilibration of the $I^{*}$ spin with the $I$-spin bath during each mixing step $\left(\tau_{M}<<T_{d p}\right.$ and $\left.T_{d f}\right)$ :

$\left\langle S_{z}\right\rangle\left(t_{c p}+N \tau_{M}\right)=1-\exp \left(-R_{d f} t_{c p}\right)\left[\frac{1}{2}+\frac{1}{2} \exp \left(-\frac{R_{d p} t_{c p}}{2}\right) \cos \left(\sum_{k=0}^{N} \Phi\left(\tau_{k}, \tau_{k}+t_{c p} / n\right)\right)\right]$. 
(ii) «Quasi- » re-equilibration of the $I^{*}$ spin with the $I$-spin bath during each mixing step $\left(T_{d p}<<\tau_{M}<<T_{d f}\right)$ :

$$
\left\langle S_{z}\right\rangle\left(t_{c p}+N \tau_{M}\right)=1-\exp \left(-R_{d f} t_{c p}\right)\left[\frac{1}{2}+\frac{1}{2} \exp \left(-\frac{R_{d p} t_{c p}}{2}\right) \prod_{k=0}^{N} \cos \left(\Phi\left(\tau_{k}, \tau_{k}+t_{c p} / n\right)\right)\right] .
$$

(iii) Full re-equilibration of the $I^{*}$ spin with the $I$-spin bath during each mixing step $\left(\tau_{M} \gg T_{d f}\right):$

$$
\left\langle S_{z}\right\rangle\left(t_{c p}+N \tau_{M}\right)=1-\exp \left(-R_{d f} t_{c p}\right) \prod_{k=0}^{N}\left[\frac{1}{2}+\frac{1}{2} \exp \left(-\frac{R_{d p} t_{c p}}{2 n}\right) \cos \left(\Phi\left(\tau_{k}, \tau_{k}+t_{c p} / n\right)\right)\right] .
$$

The integrated phase $\Phi\left(t_{0}, t_{1}\right)$ in Eqs (24-26) contains the spatial part of the $I^{*}-S$ interaction :

$$
\begin{aligned}
\Phi\left(\tau_{k}, \tau_{k}+t_{c p} / n\right)= & \int_{\tau_{k}}^{\tau_{k}+t_{c p} / n} b(t) d t \\
& =\frac{\delta_{ \pm 1}}{\omega_{r}}\left\{\sin \left[\gamma+\omega_{r}\left(\tau_{k}+\frac{t_{c p}}{n}\right)\right]-\sin \left[\gamma+\omega_{r} \tau_{k}\right]\right\} \\
& +\frac{\delta_{ \pm 2}}{2 \omega_{r}}\left\{\sin \left[2 \gamma+2 \omega_{r}\left(\tau_{k}+\frac{t_{c p}}{n}\right)\right]-\sin \left[2 \gamma+2 \omega_{r} \tau_{k}\right]\right\}
\end{aligned}
$$

where the dipolar coupling elements are defined by

$$
\begin{aligned}
& \delta_{ \pm 1}=\frac{D_{I S}}{2} \sqrt{2} \sin 2 \beta, \\
& \delta_{ \pm 2}=-\frac{D_{I S}}{2} \sin ^{2} \beta .
\end{aligned}
$$

When each CP step duration is fixed at one-half a rotation period $\left(t_{c p} / n=T_{r} / 2\right)$ the following integrated phase is obtained : 


$$
\Phi\left(\tau_{k}, \tau_{k}+T_{r} / 2\right)=\Phi_{\omega_{r}}\left(\tau_{k}\right)=-\frac{D_{I S}}{\omega_{r}} \sqrt{2} \sin 2 \beta \sin \left(\gamma+\omega_{r} \tau_{k}\right)
$$

with $\tau_{k}=k\left(\frac{T_{r}}{2}+\tau_{M}\right)$.

Clearly, MC-CP pulse sequences are then expected to recouple the $\omega_{r}$-modulated spatial part of dipolar Hamiltonian ( $c f$. Eq. (19)) [61]. Moreover, if the mixing time $\tau_{M}$ is set to a half-integer multiple of $T_{r}$ in a rotor-synchronized MC-CP pulse sequence we have $\Phi_{\omega_{r}}\left(\tau_{k}\right)=\Phi_{\omega_{r}}(0)$ so that all dephasing phases in Eqs. (24)-(26) are identical. On the other hand, if $\tau_{M}$ is set to an integral number of $T_{r}$ the absolute values of all dephasing phases are equal, i.e., $\Phi_{\omega_{r}}\left(\tau_{k}\right)= \pm \Phi_{\omega_{r}}(0)$. In fact, the recoupling CP technique SAP developed by Pratima and Ramanathan [14] may be considered as a special MC-CP pulse sequence for which there is no significant reequilibration of the $I^{*}$ spin with the $I$-spin bath during each mixing step (case (i)), i.e., $t_{c p} / n+\tau_{M}=T_{r}<<T_{d p}, T_{d f}$. In particular, when $t_{c p} / n=\tau_{M}=T_{r} / 2$, Eq. (24) yields

$$
\begin{aligned}
\left\langle S_{z}\right\rangle\left(t_{c p}+N T_{r} / 2\right) & =1-\exp \left(-R_{d f} t_{c p}\right)\left[\frac{1}{2}+\frac{1}{2} \exp \left(-\frac{R_{d p} t_{c p}}{2}\right) \cos \left(n \Phi_{\omega_{r}}(0)\right)\right] \\
= & 1-\exp \left(-R_{d f} t_{c p}\right)\left[\frac{1}{2}+\frac{1}{2} \exp \left(-\frac{R_{d p} t_{c p}}{2}\right) \cos \left(\frac{n D_{I S} T_{r}}{2 \pi} \sqrt{2} \sin 2 \beta \sin \gamma\right)\right] .
\end{aligned}
$$

Hence, when the mixing time is sufficiently short, the rotor-synchronized MC-CP (or SAP) sequence achieves efficient polarization transfer at the original centerband $\left(n_{H H}\right.$ $=0) \mathrm{HH}$ condition by a «REDOR-type » dipolar recoupling [61; 62]. In powders, the oscillations with different frequencies will destructively interfere with each other. A quasi-equilibrium state is then rapidly established yielding the $S$-spin polarization value $\overline{\left\langle S_{z}\right\rangle_{q e}}=1 / 2$, i.e., only half of the initial polarization of the $I$ spin is transferred to the $S$ spin in an isolated $I-S$ spin pair. 
The MC-CP pulse sequence is usually performed with longer mixing times [27; $28 ; 34 ; 35 ; 36 ; 37 ; 38 ; 63]$. Using $t_{c p} / n=T_{r} / 2$ and assuming that full re-equilibration of the $I^{*}$ spin with the $I$-spin bath is achieved during each mixing step (case (iii)), Eq. (26) yields

$$
\left\langle S_{z}\right\rangle\left(t_{c p}+N \tau_{M}\right)=1-\exp \left(-R_{d f} t_{c p}\right) \prod_{k=0}^{N}\left[\frac{1}{2}+\frac{1}{2} \exp \left(-\frac{R_{d p} t_{c p}}{2 n}\right) \cos \left[\Phi_{\omega_{r}}\left(\tau_{k}\right)\right],\right.
$$

where $\Phi_{\omega_{r}}\left(\tau_{k}\right)$ is given by Eq. (30).

Eq. (32) shows that the amplitude of the slow incoherent stage of the MC-CP dynamics equal to $(1 / 2)^{n}$ is then expected to strongly decrease with the number $n$ of $\mathrm{CP}$ steps so that the equilibrium state polarization reached in $\mathrm{MC}-\mathrm{CP}, \overline{\left\langle S_{z}\right\rangle_{q e}}(n)=1-$ $(1 / 2)^{n}$, is higher than the one given by the SAP sequence, $\overline{\left\langle S_{z}\right\rangle_{q e}}=1 / 2$. However, if $\tau_{M}$ is set to a half-integer multiple or an integral number of rotor periods we have already noted that $\Phi_{\omega_{r}}\left(\tau_{k}\right)= \pm \Phi_{\omega_{r}}(0)$. In this case, Eq. (32) becomes

$$
\left\langle S_{z}\right\rangle\left(t_{c p}+N \tau_{M}\right)=1-\exp \left(-R_{d f} t_{c p}\right)\left[\frac{1}{2}+\frac{1}{2} \exp \left(-\frac{R_{d p} t_{c p}}{2 n}\right) \cos \left[\Phi_{\omega_{r}}(0)\right]\right]^{n} .
$$

In such a rotor-synchronized MC-CP experiment, all cosine arguments are equal and the expansion of the product in Eq. (33) leads to many purely exponential (incoherent) terms decreasing significantly the quasi-equilibrium state polarization as in the case of a static sample [38]. Rotor-asynchronous $\mathrm{MC}-\mathrm{CP}$ is then expected to yield higher gains in magnetization transfer than rotor-synchronized MC-CP.

When the spinning frequency $\omega_{r}$ exceeds both the $I-I$ and $I-S$ dipolar interactions, it is well known that the single $\mathrm{HH}$ condition splits into a series of new 
"sideband" matching conditions $\Delta=\omega_{1 I}-\omega_{1 S}=n_{H H} \omega_{r}$ and efficient CP is obtained only at the first- and second-order sidebands $\left(n_{H H}= \pm 1, \pm 2\right)[8 ; 9]$. At the $n_{H H}= \pm 1$ and \pm 2 matching conditions, a secular (time-independent) flip-flop term exists because of the destructive interference between the modulation of the dipolar coupling by MAS and the RF fields. The zeroth-order average Hamiltonian $H_{I S}^{(0)}\left(n_{H H}\right)$ obtained by a transformation to the rotating frame of the nearest-resonant Fourier component of the time-dependent dipolar coupling $b(t)$ yields a simple analytical expression of the $\mathrm{CP}$ dynamics around a sideband matching condition which is valid under fast MAS conditions $\left(\omega_{r}>>D_{I S}\right)$ [12; 64]. Indeed, by substitution of $H_{I S}^{(0)}\left(n_{H H}\right)$ into Eq. (20), it is readily shown that the transferred polarization evolves at the $n_{H H}= \pm 1, \pm 2$ matching conditions as

$$
\left\langle S_{z}\right\rangle\left(t_{c p}\right)=1-\exp \left(-R_{d f} t_{c p}\right)\left[\frac{1}{2}+\frac{1}{2} \exp \left(-\frac{R_{d p} t_{c p}}{2}\right) \cos \left(\frac{\delta_{n_{H H}} t_{c p}}{2}\right)\right],
$$

where $\delta_{ \pm 1}$ and $\delta_{ \pm 2}$ are given by Eqs (28) and (29).

In principle, the $n_{H H}= \pm 1$ matching condition is preferred because of its larger recoupling efficiency. By contrast to the case of the MC-CP (or SAP) sequence, the recoupled heteronuclear signal is « $\gamma$-encoded » (it does not depend on the angle $\gamma$ ) $[61 ; 65]$. However, as in the case of the SAP sequence, Eq. (34) shows that $\overline{\left\langle S_{z}\right\rangle_{q e}}=$ $1 / 2$.

Since $H_{I S}^{(0)}\left(n_{H H}\right)$ is time-independent the MC-CP signal at the $n_{H H}= \pm 1$ and \pm 2 sideband conditions is similar to the one obtained under static conditions. Therefore, in the case of full re-equilibration during $\tau_{M}$, we obtain [38]

$$
\left\langle S_{z}\right\rangle\left(t_{c p}+N \tau_{M}\right)=1-\exp \left(-R_{d f} t_{c p}\right)\left[\frac{1}{2}+\frac{1}{2} \exp \left(-\frac{R_{d p} t_{c p}}{2 n}\right) \cos \left(\frac{\delta_{n_{H H}} t_{c p}}{2 n}\right)\right]^{n} .
$$



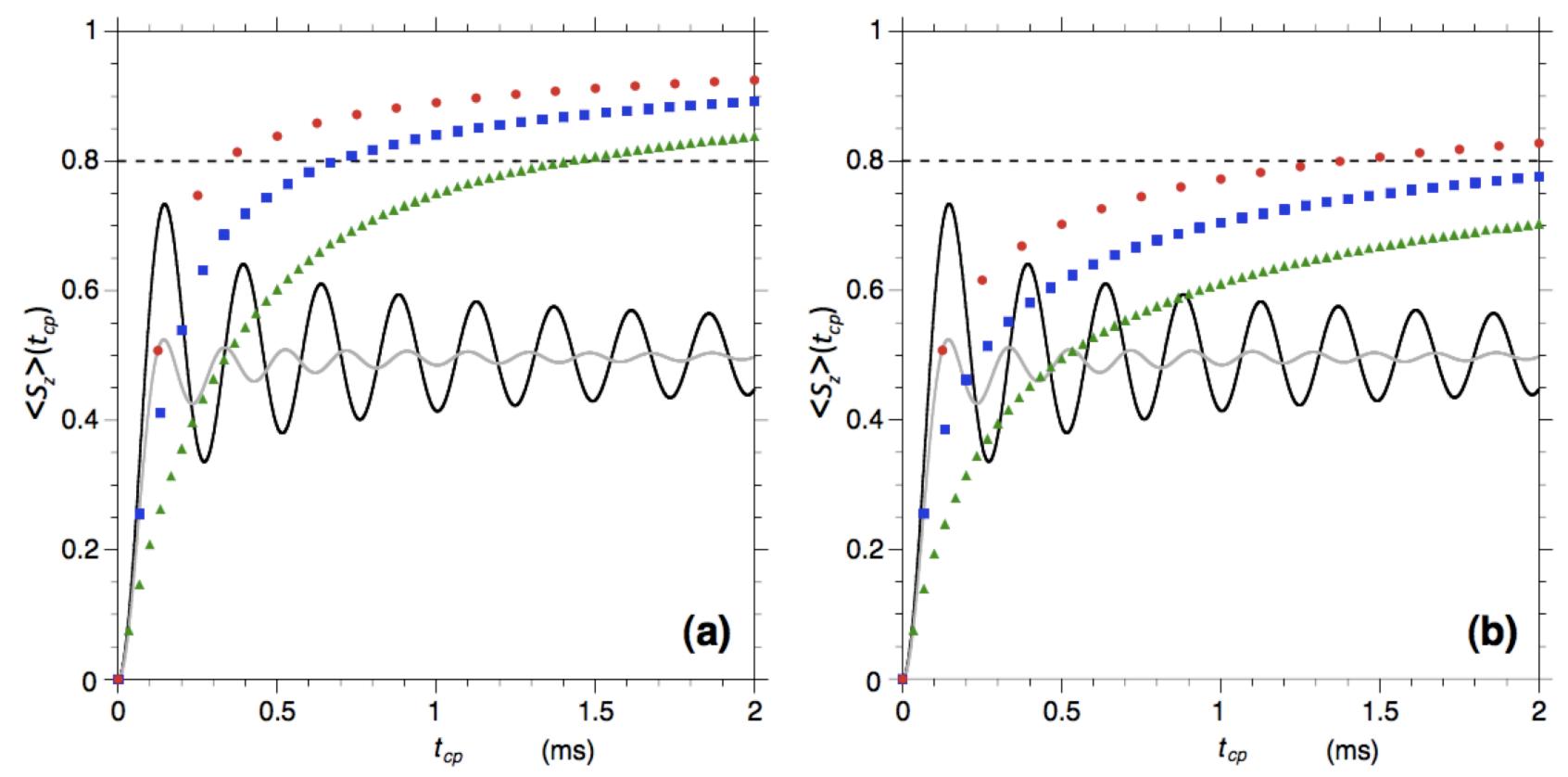

Figure 2 : Calculated polarization evolution of $S$-spin magnetization $\left\langle S_{z}\right\rangle(t)$ as a function of the total contact time $t_{c p}$ for SAP (Eq. $31:$ the SAP dynamics at any MAS rate lie on this curve) (grey line), HHCP $n_{H H}=1$ (Eq. $34: \omega_{r} \gg D_{I S}$ ) (black line) and MC-CP $n_{H H}=0$ (Eqs 32 or 33) at $v_{r}=4 \mathrm{kHz}$ (red circles), $7.5 \mathrm{kHz}$ (blue squares), $15 \mathrm{kHz}$ (green triangles). (a) Rotor-asynchronous MC-CP ; (b) Rotor-synchronized MC-CP. These calculations which assume that $t_{c p} / n=T_{r} / 2$ and $T_{d p}, T_{d f}>>t_{c p}\left(\tau_{M}>>\right.$ $T_{d f}$ for MC-CP) use an $I^{*}-S$ internuclear distance $r=1.09 \mathrm{~A}$ and take into account the partial average of the $I^{*}-S$ dipolar interaction due to the fast uniaxial rotational motion of the $\mathrm{C}_{5} \mathrm{H}_{5}$ rings of ferrocene.

Fig. 2 reports theoretical calculations of $\mathrm{CP}$ dynamics for SAP, $\operatorname{HHCP}\left(n_{H H}= \pm 1\right)$ and the MC-CP $\left(n_{H H}=0\right)$ sequences using Eqs (31) - (34). As expected, the $\gamma$-encoded HHCP dynamics has a more pronounced time-domain oscillatory behavior than the REDOR-type SAP build-up curve [66]. As a consequence, the ideal transfer efficiency of HHCP at the sideband $\mathrm{HH}$ condition reaching a maximum value of $\sim 0.73$ at short contact time is significantly higher than in the case of SAP $(\sim 0.55)$ (Fig. 2). However, for both the $\operatorname{SAP}\left(n_{H H}=0\right)$ and $\operatorname{HHCP}\left(n_{H H}= \pm 1\right.$ or \pm 2$)$ sequences, only half of the initial polarization of the $I$ spin is expected to be transferred to the $S$ spin in a fairly isolated $I-S$ spin pair after initial transient oscillations. By contrast, the MC-CP scheme with $t_{c p} / n=T_{r} / 2$ leads to a monotonous increase of polarization 
enhancing the transfer efficiency to approach almost unity, especially at moderate spinning speed ( $v_{r}=4$ and $7.5 \mathrm{kHz}$ ) (Fig. 2). Moreover, as expected from our theoretical analysis, higher intensity gains are obtained with asynchronous MC-CP. Fortunately, in practice, long mixing times $\left(\tau_{M}>>T_{r}\right)$ always are asynchronous with MAS since rotor-synchronization then necessitates the use of a rotor triggering signal by optical detection of the rotor position [67].

\subsubsection{Gaussian-powder non-secular solutions}

Simple analytical expressions of HHCP and MC-CP dynamics have been obtained above using the $I-I^{*}-S$ model. However, these (secular) solutions are valid only when the $I^{*}-S$ dipolar coupling constant is much higher than the SD rate constants. Moreover, they are restricted to the two specific situations where either the centerband $\mathrm{HH}$ matching condition is exactly fulfilled or the spinning frequency is much higher than the $I^{*}-S$ dipolar coupling constant. Therefore, it is of interest to obtain NS solutions to the QM master equation for arbitrary values of the applied RF fields and the spinning frequency. Since the time dependence of $b(t)$ can lead to spectacularly complex analytical solutions appropriate mathematical approximations must be made. A particularly attractive method is the so-called memory function (MF) approach $[50 ; 54 ; 68 ; 69 ; 70 ; 71]$ which has been used extensively in Mehring's book [4] to describe cross-polarization and spin-decoupling processes. The MF enters the theory as a consequence of the mathematical formalism, but it has the physical significance of a local-field correlation function. Considering only the Ising term of the $I-I^{*}$ dipolar interaction $\left(R_{d f}=0\right)$ in the QM master equation defined by Eqs (13) and (16) (FF approximation), it has been previously shown that the expectation value $\left\langle S_{z}\right\rangle(t)=\left\langle I_{z}^{\Sigma}\right\rangle(t)-\left\langle I_{z}^{\Delta}\right\rangle(t)$ satisfies the following integro-differential equation [54; $55 ; 71]$ 


$$
\begin{aligned}
\frac{d\left\langle I_{z}^{\chi}\right\rangle(t)}{d t} & =-\int_{0}^{t} K_{\chi}\left(t, t^{\prime}\right)\left\langle I_{z}^{\chi}\right\rangle\left(t^{\prime}\right) d t^{\prime} \\
& =-\int_{0}^{t} b(t) b\left(t^{\prime}\right) \cos \left[\chi\left(t-t^{\prime}\right)\right] \exp \left[-R_{d p}\left(t-t^{\prime}\right)\right]\left\langle I_{z}^{\chi}\right\rangle\left(t^{\prime}\right) d t^{\prime}
\end{aligned}
$$

where $\chi=\Delta$ or $\Sigma ; \Delta=\omega_{1 I}-\omega_{1 S}$ and $\Sigma=\omega_{1 I}+\omega_{1 S}$.

$K_{\chi}\left(t, t^{\prime}\right)$ is the MF which relates the rate of change of $\left\langle I_{z}^{\chi}\right\rangle$ at one time to values of $\left\langle I_{z}^{\chi}\right\rangle$ at earlier times $\left(t^{\prime} \leq t\right)$. The merit of this approach lies in the fact that $\left\langle I_{z}^{\chi}\right\rangle(t)$ is less sensitive to approximations on $K_{\chi}\left(t, t^{\prime}\right)$ than similar approximations made on $\left\langle I_{z}^{\chi}\right\rangle(t)$ itself. Note that the QM master equation (Eq. (13)) operating in a multidimensional subspace of the Liouville space has been reduced to a single differential equation (Eq. (36)). This simplification has important consequences for the treatment of spin evolution under a time-dependent coupling $b(t)$ allowing analytical calculations without worrying about non-commutativity and time-ordering [54]. Moreover, the effect of the XY or planar term (of rate constant $R_{d f}$ ) may be taken into account since the following 'semi'-NS (SNS) solution is valid when $\Sigma>>$ $D_{I S} \gg R_{d f}[55]$

$$
\left\langle S_{z}\right\rangle\left(t_{c p}\right)=1-\exp \left(-R_{d f} t_{c p}\right)\left[1 / 2+\left\langle I_{z}^{\Delta}\right\rangle\left(t_{c p}\right)\right]
$$

The remaining part of the calculation requires solving Eq. (36) with $\chi=\Delta$. However, an exact calculation of $\left\langle I_{z}^{\Delta}\right\rangle(t)$ from Eq. (36) is difficult to obtain even in the case of an isolated $I-S$ spin pair $\left(R_{d p}^{I}=0\right)$ [54]. A first simplification is obtained by remarking that only the terms involving the time difference $t-t^{\prime}=\tau$ remain in the MF $K_{\Delta}\left(t, t^{\prime}\right)$ after powder averaging over the product $b(t) b\left(t^{\prime}\right)$. Assuming that other terms only lead to minor interference effects, $K_{\Delta}(\tau)$ in a powder CPMAS experiment is given by $[50 ; 54]$

$$
K_{\Delta}(\tau)=M_{2}^{I S} K_{M A S}(\tau) \cos (\Delta \tau) \exp \left(-R_{d p} \tau\right),
$$

where $M_{2}^{I S}=D_{I S}^{2} / 5$ is the second moment of the $I^{*}-S$ dipolar interaction and 


$$
K_{M A S}(\tau)=\frac{2}{3} \cos \left(\omega_{r} \tau\right)+\frac{1}{3} \cos \left(2 \omega_{r} \tau\right) .
$$

The Anderson-Weiss (AW) approximation $[4 ; 47 ; 50 ; 72 ; 73]$ may then be applied to derive a simple analytical solution to Eq. (36) :

$$
\frac{d}{d t}\left\langle I_{z}^{\Delta}\right\rangle(t)=-\int_{0}^{t} K_{\Delta}(\tau)\left\langle I_{z}^{\Delta}\right\rangle(t-\tau) d \tau \approx-\left\langle I_{z}^{\Delta}\right\rangle(t) \int_{0}^{t} K_{\Delta}(\tau) d \tau
$$

Substituting Eq. (38) into Eq. (40), one finds the solution $\left\langle I_{z}^{\Delta}\right\rangle(t)=\left\langle I_{z}^{\Delta}\right\rangle(0) G_{\Delta}(t)$ where $[73 ; 74]$

$$
\begin{aligned}
G_{\Delta}(t) & =\exp \left[-\int_{0}^{t}(t-\tau) K_{\Delta}(\tau) d \tau\right] \\
& =\exp \left\{M_{2}^{I S}\left[\begin{array}{l}
\frac{1}{3} f\left(R_{d p}, \Delta-\omega_{r}, t\right)+\frac{1}{3} f\left(R_{d p}, \Delta+\omega_{r}, t\right) \\
+\frac{1}{6} f\left(R_{d p}, \Delta-2 \omega_{r}, t\right)+\frac{1}{6} f\left(R_{d p}, \Delta+2 \omega_{r}, t\right)
\end{array}\right]\right\} .
\end{aligned}
$$

The $f(x, \omega, t)$ function is defined in Refs [73; 74]. Eq. (41) is a very useful result, applicable to a wide range of dynamical situations. Indeed, Cobo et al. [75] have successfully used Eq. (41) considering that $K_{\Delta}(\tau)$ describes the local fluctuations in the $I-S$ dipolar field to study the combined effects of sample spinning and molecular motion on Lee-Goldburg CP experiments. In this case, motional exchange dominates over spin exchange so that $R_{d p}$ is replaced by the probability of reorientation per unit of time $1 / \tau_{C}$.

For HHCP, $\left\langle I_{z}^{\Delta}\right\rangle(0)=1 / 2$, and Eq. (37) yields

$$
\left\langle S_{z}\right\rangle\left(t_{c p}\right)=1-\exp \left(-R_{d f} t_{c p}\right)\left[\frac{1}{2}+\frac{1}{2} G_{\Delta}\left(t_{c p}\right)\right],
$$

where $G_{\Delta}\left(t_{c p}\right)$ is given by Eq. (41). 

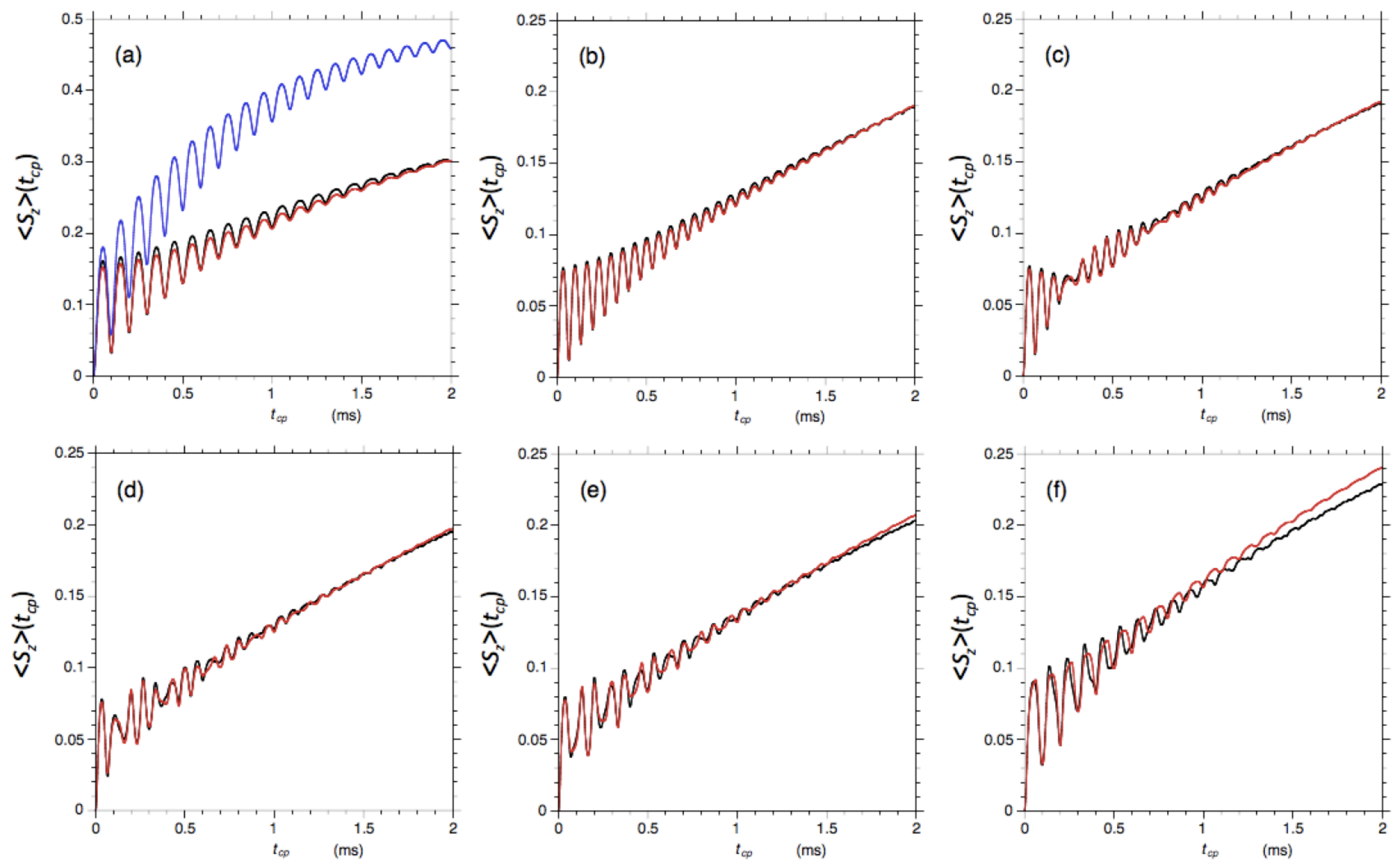

Figure 3 : Polarization evolution of $S$-spin magnetization $\left\langle S_{z}\right\rangle(t)$ as a function of the HHCP contact time under MAS obtained by numerical integration of the QM master equation, Eq. (13) (black line), the MKBE-MAS solution, Eq. (21) (blue line), and the NS AW solution, Eq. (42) (red line), for an Ising SE interaction ( $T_{d p}=0.4 \mathrm{~ms}$; $\left.R_{d f}=0\right):$ (a) $v_{r}=10 \mathrm{kHz}\left(S_{f}=1.3875\right)$ at the centerband $\mathrm{HH}$ condition $(\Delta=0) ; v_{r}=$ $15 \mathrm{kHz}\left(S_{f}=1.15\right.$ ) with $\Delta / 2 \pi=$ (b) 0 , (c) 1 , (d) 2 , (e) 3 , (f) $5 \mathrm{kHz}$. The AW calculations use $1 / \sqrt{M_{2}^{I S}}=30.67 \mu \mathrm{s}$. The other parameters are the same as in Fig. 2.

Fig. 3 compares HHCP buildup curves obtained by using this NS AW solution, Eq. (42), the (secular) MKBE-MAS solution, Eq. (21), and exact numerical integration of the QM master equation, Eq. (13), for an Ising SE interaction ( $\left.R_{d f}=0\right)$ at $v_{r}=10$ and $15 \mathrm{kHz}$. It is first remarked that the MKBE-MAS solution gives a poor agreement with the numerical calculations although both calculations exhibit strong rotational echoes due to the inhomogeneous character of the $I^{*}-S$ spin pair interaction (Fig. 3(a)). Since Eq. (21) is an exact solution to the QM master equation in the absence of spin diffusion ( $R_{d p}=R_{d f}=0$ ) this discrepancy must be attributed to the 
effect of NS terms. Hence, by contrast to the case of a slowly rotating sample [55], the secular approximation at the centerband $\mathrm{HH}$ condition is no longer valid in the presence of significant coherent averaging by MAS, i.e., when $\omega_{r}$ becomes comparable to $D_{I S}$. On the other hand, Fig. 3 clearly shows that exact numerical calculations of the HHCP dynamics are very well reproduced by the approximate analytical solution based upon the AW theory or Gaussian frequency distribution model provided that a scaling factor $S_{f}$ accounting for an apparently increased time constant $T_{d p}^{A W}=S_{f} \times T_{d p}$ is considered. $S_{f}$ only depends on the ratio $x=M_{2}^{I S} / \omega_{r}^{2}$. Fig. 3 demonstrates that Eq. (42) gives a very good agreement with exact numerical calculations in the strong collision limit $\left(\omega_{r} T_{d p} \gg 1\right)[76]$ at short and intermediate contact times $\left(t_{c p}<\sim 5 T_{d p}\right)$, even for mismatched HH conditions $(\Delta \neq 0)$. This result clearly confirms that the AW approach is not limited to self-commuting MAS Hamiltonian (super)operators. Simulations as those shown in Fig. 3 were performed for various second moment values and MAS rates to obtain the scaling factor $S_{f}$. Fig. 4 shows the variation of $S_{f}$ as a function of $x$ for $x<0.7$ where the Gaussian powder NS AW solution provides a better agreement with the numerical calculations than the exact powder MKBE-MAS solution. As expected, $S_{f}$ approaches unity when $x$ goes to zero. Note also that the agreement between the AW and the numerical calculations could be further improved by adding an extra scaling to $M_{2}^{I S}$ [77]. 


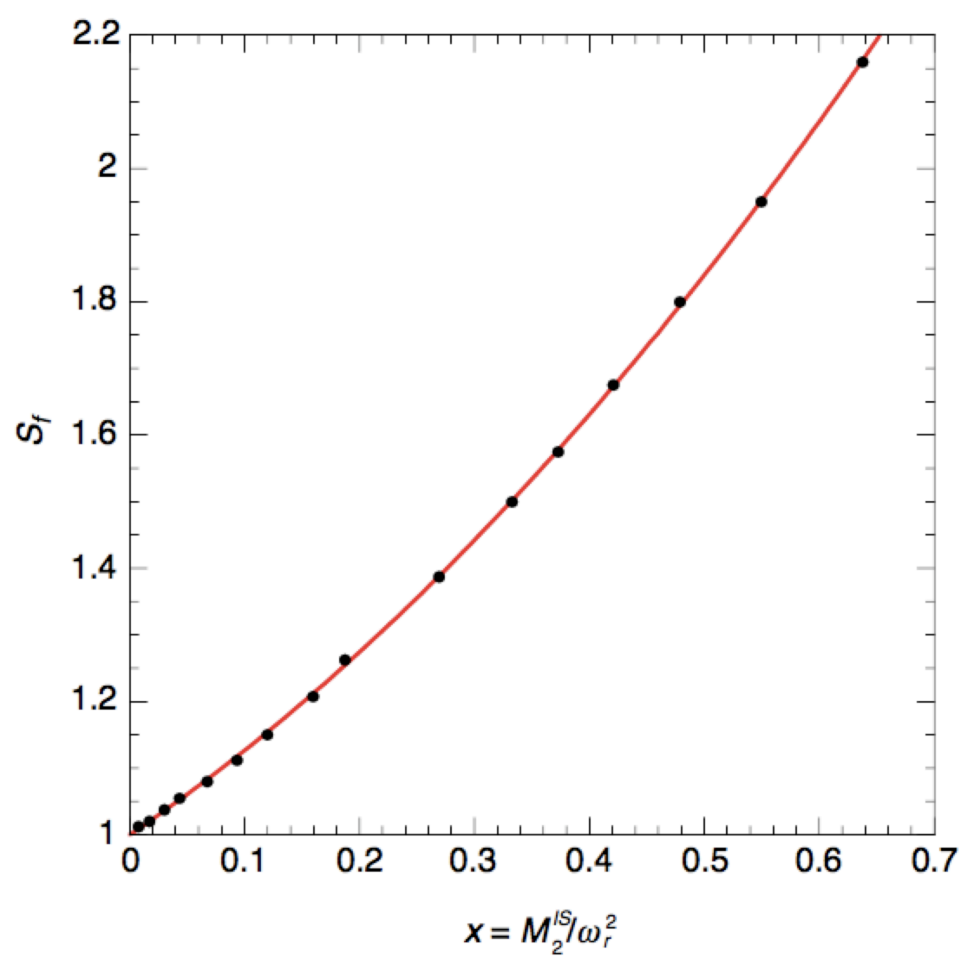

Figure 4 : Plot of the scaling factor $S_{f}$ as a function of $x=M_{2}^{I S} / \omega_{r}^{2}$ obtained by fits as illustrated in Fig. 3 using several values of $M_{2}^{I S}, T_{d p}$ and $v_{r}$. The polynomial fitting function (red line) is $S_{f}=1.00+1.16 x+1.03 x^{2}$.

In MC-CP with $\tau_{M}>>T_{d p}$ and/or $T_{d f}$, the ZQ and DQ coherences vanish during the mixing time (z-filter) [36]:

$$
\left\langle I_{x}^{\Delta}\right\rangle\left(\tau_{k}\right)=\left\langle I_{y}^{\Delta}\right\rangle\left(\tau_{k}\right)=\left\langle I_{x}^{\Sigma}\right\rangle\left(\tau_{k}\right)=\left\langle I_{y}^{\Sigma}\right\rangle\left(\tau_{k}\right)=0 .
$$

On the other hand, assuming full re-equilibration of the $I^{*}$ spin with the thermal bath of the remaining $I$ spins $\left(\tau_{M} \gg T_{d f}\right.$ ), we have $\left\langle I_{z}\right\rangle\left(\tau_{k}\right)=1$. Moreover, since the $S$ magnetization is retained during each mixing step $\left(\tau_{M}<<T_{1}^{S}\right),\left\langle S_{z}\right\rangle\left(\tau_{k+1}\right)=$ $\left\langle S_{z}\right\rangle\left(\tau_{k}+t_{c p} / n\right)$. Hence, considering only the Ising term of the $I-I^{*}$ dipolar interaction, the following recursion relation for the evolution of the $S$-spin polarization during each cycle of the MC-CP sequence is valid

$$
\left\langle S_{z}\right\rangle\left(\tau_{k+1}\right)=\left\langle I_{z}^{\Sigma}\right\rangle\left(\tau_{k}\right)-\left\langle I_{z}^{\Delta}\right\rangle\left(\tau_{k}\right) G_{\Delta}\left(t_{c p} / n\right)
$$




$$
=\frac{1}{2}\left\{\left\langle S_{z}\right\rangle\left(\tau_{k}\right)+1+\left[\left\langle S_{z}\right\rangle\left(\tau_{k}\right)-1\right] G_{\Delta}\left(t_{c p} / n\right)\right\} .
$$

Eq. (44) then yields the following SNS solution for the $S$-spin polarization at the end of the MC-CP sequence

$$
\left\langle S_{z}\right\rangle\left(t_{c p}+N \tau_{M}\right)=1-\exp \left(-R_{d f} t_{c p}\right)\left[\frac{1}{2}+\frac{1}{2} G_{\Delta}\left(t_{c p} / n\right)\right]^{n} .
$$
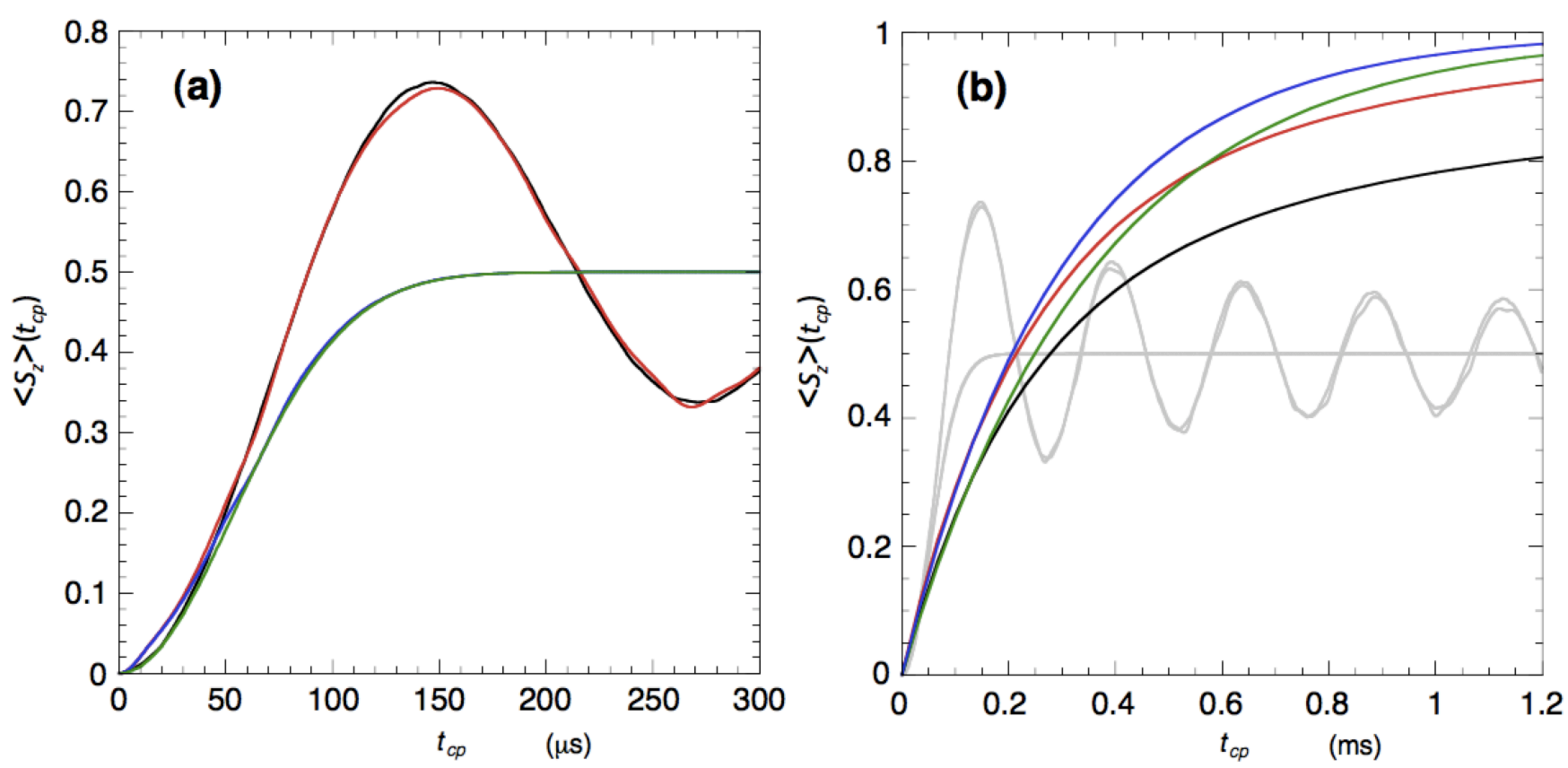

Figure 5 : $\mathrm{CP}$ dynamics at the $n_{H H}= \pm 1$ sideband HH condition : (a) HHCP and (b) MC-CP $\left(t_{c p} / n=33.33 \mu \mathrm{s}\right)$ exact calculation of isotropic powder obtained by the independent sideband approximation (black line), Eqs (a) (34) and (b) (35), by numerical integration of the QM master equation (Eq. (13)) at $v_{r}=15 \mathrm{kHz}$ (red line), by the Gaussian powder (AW) model, (a) Eq. (42) and (b) Eq. (45), in the fast MAS limit approximation $\left(\omega_{r}^{2}>>M_{2}^{I S}\right)$ (green line) and at $v_{r}=15 \mathrm{kHz}$ (blue line). For comparison, the HHCP dynamics calculations of (a) are reproduced in (b) by grey lines. Both the exact and Gaussian powder calculations use $R_{d p}=R_{d f}=0$. The other parameters are the same as in Fig. 3. 
As expected, this result is similar to the ones obtained in the secular approximation at the centerband (Eqs (32) and (33)) and sideband (Eq. (35)) HH matching conditions. However, in the AW approach, all orientational correlations between the CP steps of the $\mathrm{MC}-\mathrm{CP}$ sequence are neglected. This is the reason why the rotor-synchronization effects shown in Fig. 2 cannot be reproduced when using Eq. (45). The MC-CP transfer efficiency is then generally expected to be overestimated by the AW model. In Fig. 5, we compare the HHCP and MC-CP dynamics at the $n_{H H}= \pm 1 \mathrm{HH}$ matching condition using the Gaussian powder approximation [74] with analytical and numerical exact powder calculations. As expected, in the fast MAS limit $\left(\omega_{r} \gg\right.$ $D_{I S}$ ), Eqs (34) and (35) give results that are in excellent agreement with those obtained by numerical integration of the QM master equation. Moreover, Fig. 5 shows that the transient oscillations during HHCP on a sideband matching condition stay almost unchanged when the spinning frequency $\left(v_{r}=15 \mathrm{kHz}\right)$ approaches the dipolar coupling ( $D_{I S} / 2 \pi=11.6 \mathrm{kHz}$ ) so that Eq. (34) still gives a good approximation of the HHCP dynamics. A closer inspection of the CP dynamics at short contact times $\left(t_{c p}<\sim 50 \mu \mathrm{s}\right)$ nevertheless shows that the initial part of HHCP dynamics at $v_{r}=15 \mathrm{kHz}$ departs significantly from the HHCP buildup in the fast MAS limit approximation (Fig. 5(a)). The AW or Gaussian powder approximation leads to a monotonous curve without the transient oscillations following the average time course of the exact powder calculations [74]. A very good agreement between the Gaussian and exact powder calculations is nevertheless observed at short contact times for $v_{r}=15 \mathrm{kHz}$ as well as in the fast MAS limit approximation (Fig. 5(a)). This behavior is explained considering that, at short contact time, the CP transfer only depends on the second and not the higher moments of the frequency distribution. The slight difference between the short contact time CP dynamics in the fast MAS limit approximation and at $v_{r}=15 \mathrm{kHz}$ has important consequences for MC-CP. Indeed, the numerical calculations of Fig. 5(b) demonstrate that the MC-CP dynamics at $v_{r}=15 \mathrm{kHz}$ with $t_{c p} / n=T_{r} / 2=33.33 \mu$ s can no longer be approximated by Eq. (35), the transfer efficiency being underestimated by the calculation in the fast MAS 
limit approximation. Remarkably, Fig. 5(b) shows that the AW approach accounts for this difference and gives a good agreement with exact numerical calculations at short contact times $\left(t_{c p}<\sim 200 \mu \mathrm{s}\right)$. Clearly, these results further demonstrate that, although not giving rigorously correct results at longer contact times, the strength of the AW model is to fit well the exact powder calculation at short evolution times [74]. We have previously shown that HHCP matching profiles taken at short contact times can be very well reproduced by AW calculations [74]. Since the MC-CP sequence is usually performed using short $\mathrm{CP}$ contacts of duration $t_{c p} / n$ of a few tens of microseconds MC-CP matching profiles are expected to be well described by the Gaussian powder model. Indeed, as shown by Fig. 6(a,b), the agreement between the exact and Gaussian powder MC-CP matching profiles is good over the whole range of contact times except from a slight overestimation of transfer efficiency at long contact times. More importantly, Fig. 6(c,d,e) shows that the MC-CP matching conditions are much broader than the HHCP matching conditions at contact times longer than $\sim 1 / D_{I S}$ and that the MC-CP transfer efficiency is maximum between the first- and the second-order sideband matching conditions $(\Delta / 2 \pi \approx \pm 20 \mathrm{kHz})$. Sideband matching is difficult for HHCP at contact times longer than $\sim 200 \mu \mathrm{s}$ because of the excessive sensitivity of the matching condition to the RF-field amplitudes of the two channels [78]. MC-CP is then expected to be much more tolerant than HHCP to $\mathrm{HH}$ mismatch, RF-field inhomogeneity and fluctuations in both RF power and the spinning speed. 

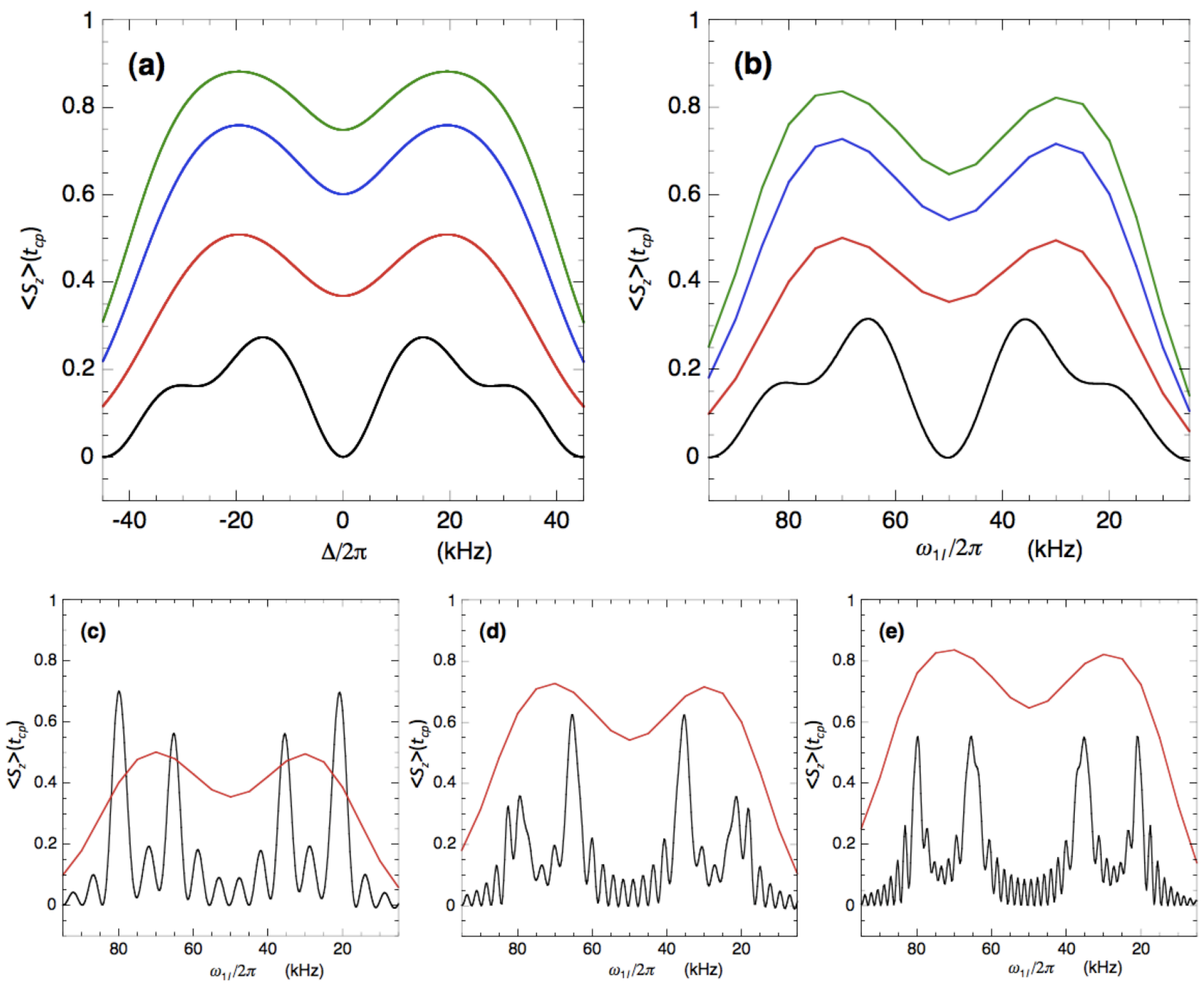

Figure 6 : $\mathrm{CP}$ matching profiles at $v_{r}=15 \mathrm{kHz}$ for different contact times. (a) Gaussian powder (AW) model and (b) exact powder calculations by numerical integration of the QM master equation $\left(\omega_{1 S} / 2 \pi=50 \mathrm{kHz}\right)$ for HHCP with $t_{c p}=67$ $\mu$ s (black line) and for MC-CP with $t_{c p}=0.2 \mathrm{~ms}$ (red line), $0.4 \mathrm{~ms}$ (blue line) and 0.6 ms (green line). Exact powder calculations of HHCP (black line) and MC-CP (red line) matching profiles for $t_{c p}=$ (c) $0.2 \mathrm{~ms}$, (d) $0.4 \mathrm{~ms}$ and (e) $0.6 \mathrm{~ms}$. The other parameters are the same as in Fig. 5.

\subsection{Memory (or non-Markoffian) effects of the I-spin bath}

The flip-flop spin motion gets slow when MAS averages out the dipolar couplings $d_{j k}$. In other words, the correlation time of the $I$-spin bath $\tau_{x}$ increases with increasing spinning rate so that the Markoff or FF approximation $\left(d_{S E} \tau_{x}<<1\right)$ may 
no longer be valid. As a consequence, the $I-I *$ SE interaction may no longer be characterized by a simple exponential decay of rate constant $R_{d p}$ as in Eq. (38). However, neglecting cross correlations of higher order, the MF may still be approximated by the product

$$
K_{\Delta}(\tau)=M_{2}^{I S} K_{M A S}(\tau) \cos (\Delta \tau) C_{x}(\tau),
$$

where the dipolar fluctuation autocorrelation function $C_{x}(t)$ function accounts for the decoherence of the spin $I^{*}$ to its multiple-spin environment ( $I$-spin bath) due to the Ising term of the $I-I^{*}$ interaction [55]. $C_{x}(t)$ may exhibit a non-exponential decay given by the non-Markoffian character of relaxation, in which the finite duration of the MF $K_{z z}(\tau)=\overline{F_{z}(\tau) F_{z}(0)}$ (Eq. (12)) is influenced by the coherent spatial averaging process due to MAS. The corresponding semi-classical SE interaction Hamiltonian is given by Eq. (10) with $\alpha=2$ and $\beta=0$ :

$$
\hat{H}_{S E}^{\text {Ising }}(t)=2 F_{z}(t) \hat{I}_{z}
$$

Since the $I^{*}-S$ dipolar coupling constant is independent of the coordinates of the spins $I_{k}$ in the bath $C_{x}(t)$ represents the time evaluation for the free induction decay (FID) of the $I^{*}$ spins determined by $\hat{H}_{S E}^{\text {Ising }}(t)[4 ; 50 ; 70]$. Using the fact that $\hat{H}_{S E}^{\text {Ising }}(t)$ commutes with itself at all times, the FID is readily evaluated as

$$
\left\langle I_{x}\right\rangle(t)=\frac{\operatorname{Tr}\left\{\exp \left[-i \varphi(t) \hat{I}_{z}\right] \hat{I}_{x} \exp \left[i \varphi(t) \hat{I}_{z}\right] \hat{I}_{x}\right\}}{\operatorname{Tr}\left(\hat{I}_{x}^{2}\right)}=\cos \varphi(t)
$$

where

$$
\varphi(t)=2 \int_{0}^{t} F_{z}\left(t^{\prime}\right) d t^{\prime}
$$

Taking the ensemble average for a Gaussian random variable, we obtain

$$
C_{x}(t)=\overline{\left\langle I_{x}\right\rangle(t)}=\overline{\cos \varphi(t)}=\exp \left\{-\frac{1}{2} \overline{\varphi^{2}(t)}\right\}
$$

Using the property of stationarity, $C_{x}(t)$ is then related to the local-field autocorrelation function $K_{z z}(\tau)$ through the well-known AW formula [72] (cf. also Eq. (41)) 


$$
C_{x}(t)=\exp \left[-\int_{0}^{t}(t-\tau) K_{z z}(\tau) d \tau\right]
$$

Eqs (37), (40), (46) and (50) show that the knowledge of $K_{z z}(\tau)$ completely determines the CP dynamics by a two-step MF (TS-MF) approach $[4 ; 50 ; 70]$ in the AW approximation. Assuming that spin fluctuations and sample rotation are statistically independent, an ansatz for the MF $K_{z z}(\tau)$ describing the stochastic process of the fluctuations for the $z$ component $F_{z}(t)$ (Ising term) of the spins $I_{k}$ due to flip-flop transitions within the $I$-spin bath is given by [50]

$$
K_{z z}(\tau)=N_{2}^{I I} K_{M A S}(\tau) \exp \left(-\tau / \tau_{x}\right),
$$

where $N_{2}^{I I} \propto d_{S E}^{2}$ is the second moment of $C_{x}(t)$, the periodic function $K_{M A S}(t)$ is given, as before, by Eq. (39) and $\tau_{x}$ is the correlation time of the $I$-spin bath in the rotating frame. Taking into account the scaling of the homonuclear dipolar couplings by the large RF field which amounts to a factor $-1 / 2$ ( $c f$. Eq. (9)) and the reduction factor of $2 / 3$ due to truncation of the XY term $(\beta=0)$, an estimation of $N_{2}^{I I}$ is given by $M_{2}^{I I} / 9$ where $M_{2}^{I I}$ is the second moment of the FID of the $I$ spins.

By substitution of Eq. (51) into Eq. (50) and integration, an analytical solution identical to the one for the free induction decay (FID) [73] is obtained for $C_{x}(t)$ :

$$
C_{x}(t)=\exp \left\{N_{2}^{I I}\left[\frac{2}{3} f\left(1 / \tau_{x}, \omega_{r}, t\right)+\frac{1}{3} f\left(1 / \tau_{x}, 2 \omega_{r}, t\right)\right]\right\} .
$$

It is readily verified that $C_{x}(t)$ gives a simple exponential decay of rate $R_{d p}=N_{2}^{I I} \tau_{x}$ in the limit of a fast fluctuating $I$-spin bath $\left(\sqrt{N_{2}^{I I}} \tau_{x}\right.$ and $\left.\omega_{r} \tau_{x}<<1\right)$, as it must be (cf. Eqs (36) and (38)). An analytical evaluation of $\left\langle S_{z}\right\rangle(t)$ using Eq. (52) seems nevertheless practically unmanageable. However, in the strong collision limit $\left(\omega_{r} \tau_{x}\right.$ $>>1)$, it is remarked that $C_{x}(t)$ simplifies into [73] 


$$
C_{x}(t)=\exp \left\{-\frac{t}{T_{d p}\left(\omega_{r}\right)}-\frac{3 N_{2}^{I I}}{4 \omega_{r}^{2}}\left[1-\frac{1}{9}\left(8 \cos \left(\omega_{r} t\right)+\cos \left(2 \omega_{r} t\right)\right) \exp \left(-t / \tau_{x}\right)\right]\right\},
$$

where

$$
T_{d p}\left(\omega_{r}\right)=\frac{4 \omega_{r}^{2} \tau_{x}}{3 N_{2}^{I I}}
$$

Moreover, if $N_{2}^{I I} / \omega_{r}<1, C_{x}(t)$ can be developed into series. In particular, the second-order development of Eq. (53) gives

$$
C_{x}(t)=\exp \left(-\frac{t}{T_{d p}\left(\omega_{r}\right)}\right)\left\{1-\frac{3}{4} \frac{N_{2}^{I I}}{\omega_{r}^{2}}+\frac{N_{2}^{I I}}{\omega_{r}^{2}}\left[\frac{2}{3} \cos \left(\omega_{r} t\right)+\frac{1}{12} \cos \left(2 \omega_{r} t\right)\right] \exp \left(-\frac{t}{\tau_{x}}\right)\right\} .
$$

Using Eq. (55), an analytical solution (too long to be included here) is readily obtained for $\left\langle S_{z}\right\rangle(t)$ by substitution of Eq. (46) into Eq. (40) and integration. When $\omega_{r} \gg N_{2}^{I I}$, Eqs (53) and (54) clearly show that $C_{x}(t)$ decays exponentially as in the case of a fast fluctuating $I$-spin bath but its rate constant $R_{d p}\left(\omega_{r}\right)=1 / T_{d p}\left(\omega_{r}\right)$ is expected to decrease with $\omega_{r}$ and $\tau_{x}$ according to Eq. (54). This result shows that the FF approximation may still provide a good description of the spin dynamics under fast MAS using SD rate contants $R_{d p}\left(\omega_{r}\right)$ and $R_{d f}\left(\omega_{r}\right)$. However, $C_{x}(t)$ is no longer exponential when $\omega_{r}^{2}$ becomes comparable to $N_{2}^{I I}$. The main consequence is an increase of the polarization transfer at the centerband $\mathrm{HH}$ matching condition. This phenomenon can be viewed as a secondary recoupling effect that restores secular transfer for $\Delta=0$ [8]. As illustrated in Fig. 7(a), while the TS-MF calculation improves considerably the transfer efficiency at the centerband $\mathrm{HH}$ matching condition $(\Delta=0)$, the CP dynamics for $\Delta= \pm \omega_{r}, \pm 2 \omega_{r}$ which is driven by recoupling of the large $I^{*}-S$ dipolar interaction stay essentially unaffected by memory effects of the $I$-spin bath. Indeed, in agreement with previous theoretical results $[10 ; 11]$, the TS-MF analytical solution shows that polarization transfer at the $n_{H H}=0 \mathrm{HH}$ 
condition occurs due to a pseudo-heteronuclear dipolar interaction induced by the $I$ $I^{*}$ SE interaction, i.e., proportional to $M_{2}^{I S} N_{2}^{I I} / \omega_{r}^{2}$. Note that, under fast MAS (strong collision limit), the $\mathrm{CP}$ rate at the centerband $\mathrm{HH}$ condition is inversely proportional to the spinning speed and that for sideband-matching $\mathrm{CP}$ does not depend upon the MAS frequency. It should be finally pointed out that the calculation of the CP dynamics by the TS-MF approach presented above differs from the method proposed by Gerstein and coworkers [50] although the same analytical expression (Eq. (52)) is employed for $C_{x}(t)$. Indeed, Gerstein et al. [50] use the classical I-S model [6] which assumes that $C_{x}(t)$ decays rapidly when compared to the $\mathrm{CP}$ dynamics (fast correlation limit) [4; 70]. Fast correlation is based on the condition that $N_{2}^{I I}$ is large enough $\left(N_{2}^{I I} \gg M_{2}^{I S}\right)$ that the upper limit of the integral in Eq. (40) can be extended to infinity. This assumption leads to a simple exponential behavior of CP dynamics and permits the calculation of a CP rate $1 / T_{I S} \propto \int_{0}^{\infty} K_{\Delta}(\tau) d \tau$ which depends on $\Delta[50]$. By contrast, in the case of the $I-I^{*}-S$ model $\left(M_{2}^{I S} \gg N_{2}^{I I}\right)$, the decay of $C_{x}(t)$ is slower than $G_{\Delta}(t)$ so that the less severe AW approximation ( $c f$. Eq. (40)) must be applied. Moreover, in the classical $I-S$ model with a small spin population ratio, i.e., $\varepsilon=N_{S} / N_{I} \ll 1$ [6], each $S$ spin is supposed to interact to a small degree with a large number $n_{I}$ of $I$ spins $\left(n_{I} \gg 1\right)$ so that $\left(1+n_{I}\right) /\left(2 n_{I}\right) \approx 1 / 2$. This is the reason why the CP rate defined in Eq. (13) of Ref. [50] differs by a factor $1 / 2$ with the one deduced from Eq. (46) $\left(n_{I}=1\right.$ in the $I-I^{*}-S$ model) [45]. 

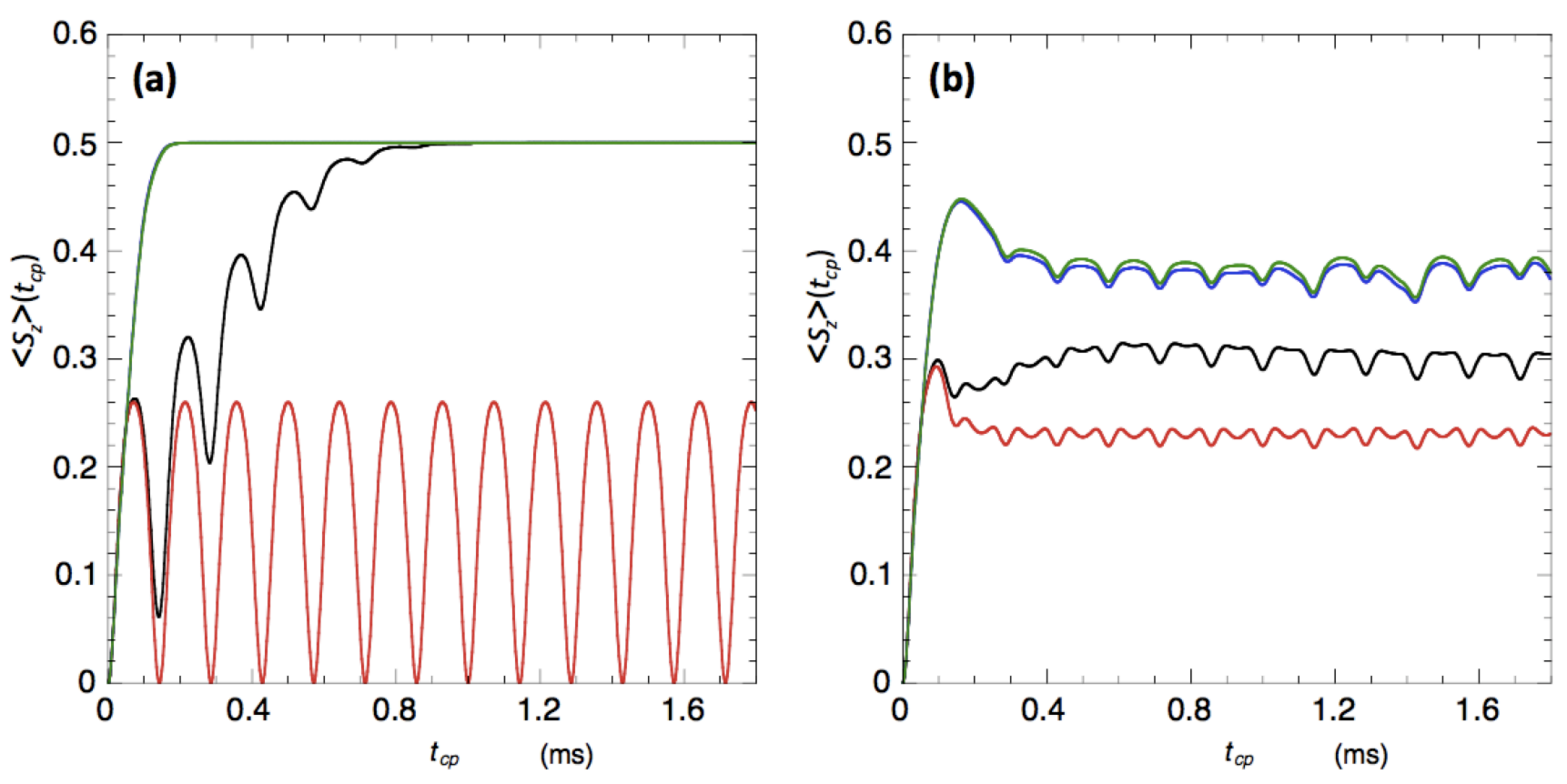

Figure 7 : Polarization evolution of $S$-spin magnetization $\left\langle S_{z}\right\rangle(t)$ as a function of the HHCP contact time under MAS $\left(v_{r}=7 \mathrm{kHz}\right)$ obtained by using the AW theory for an Ising SE interaction $\left(R_{d f}=0\right)$ : fast fluctuation approximation $\left(T_{d p}=100 \mathrm{~ms}\right)$ for $n_{H H}$ $=0$ (red line) and $n_{H H}= \pm 1$ (blue line); two-step memory function approach $\left(1 / \sqrt{N_{2}^{I I}}\right.$ $=0.1 \mathrm{~ms}$ and $\tau_{x}=100 \mathrm{~ms}$ ) for $n_{H H}=0$ (black line) and $n_{H H}= \pm 1$ (green line). (a) No $\mathrm{HH}$ mismatch distribution $\left(\sigma_{\Delta}=0\right)$; (b) $\mathrm{HH}$ mismatch distribution with $\sigma_{\Delta} / 2 \pi=3$ $\mathrm{kHz}$. All calculations use $1 / \sqrt{M_{2}^{I S}}=30.67 \mu \mathrm{s}$.

As already mentioned, RF inhomogeneity effects on $\mathrm{HHCP}$ are expected to be important. In principle, a quantitative evaluation of the effects of RF inhomogeneity would necessitate the knowledge of RF amplitudes at an arbitrary position within the sample volume. Gupta et al [79] have recently analyzed nutation spectra using a realistic model of the RF inhomogeneity profile across the sample space for singlecoil probes. In our case, however, such an analysis would require to consider the different geometries of the two RF coils of our 3.2-mm E-free probe. In the absence of such a detailed information and since the CP process in the high-field approximation only depends on the mismatch parameter $\Delta=\omega_{1 I}-\omega_{1 S}$, the main characteristics of the signal may, nevertheless, be examined by assuming a given $\mathrm{HH}$ 
mismatch distribution $P(\Delta)$. A convenient choice for $P(\Delta)$ is the Gaussian distribution defined by

$$
P(\Delta)=\frac{1}{\sigma_{\Delta} \sqrt{2 \pi}} \exp \left[\frac{-(\Delta-\bar{\Delta})^{2}}{2 \sigma_{\Delta}^{2}}\right],
$$

where $\bar{\Delta}$ and $\sigma_{\Delta}$ are, respectively, the mean value and standard deviation of $P(\Delta)$. We have previously used this HH mismatch distribution to account for the effect of $\mathrm{RF}$ inhomogeneity on experimental results of $\mathrm{CP}$ with varying contact time (CPVC) and verified that only the general shape of $P(\Delta)$ is relevant [64]. It must also be pointed out that Eq. (56) gives CPVC lineshape distortions ( $c f$. Fig. 6 of Ref. [64]) that are very similar to the ones obtained by Gupta et al [79]. Furthermore, note that these two works have led to identical conclusions about spatial selectivity of the $\mathrm{HH}$ matching condition in CPVC experiments. The influence of a mismatch distribution with $\sigma_{\Delta} / 2 \pi=3 \mathrm{kHz}$ on the HHCP dynamics of Fig. 7(a) are reported in Fig. 7(b). As expected, the strong rotational echoes observed at the centerband matching condition are severely reduced by RF inhomogeneity. However, a close inspection of Fig. 7(b) reveals that trains of rotational echoes of limited amplitude but with a very long decay time are still present. More importantly, it is seen that, despite the presence of a RF mismatch distribution, the polarization transfer efficiency for $\bar{\Delta}=0$ is still significantly increased by memory effects of the $I$-spin bath. For $\bar{\Delta}= \pm \omega_{r}$, Fig. 7(b) shows that the HH mismatch distribution results in a decrease of the quasiequilibrium polarization and the apparition of small rotational echoes as the ones observed for $\bar{\Delta}=0$ both in the FF approximation and with the TS-MF approach.

\section{Experimental}

Polycrystalline samples of ferrocene and $L$-alanine were purchased from Fluka and Sigma Chemical, respectively, and used without further purification. The NMR experiments were performed at room temperature on a Bruker Avance III 750 
spectrometer, operating at frequencies of 188.65 and $750.19 \mathrm{MHz}$ for ${ }^{13} \mathrm{C}$ and ${ }^{1} \mathrm{H}$ resonance, respectively, and equipped with Bruker CPMAS probes using 2.5 and 3.2mm-o.d. rotors. The spinning frequency was fixed at $v_{r}=4,7$ or $15 \mathrm{kHz}$. The proton and carbon RF fields calibrated directly with the ferrocene or $L$-alanine sample using a two-dimensional nutation experiment [80] were carefully adjusted to fulfill the centerband $\left(n_{H H}=0\right)$ or the first-order sideband $\left(n_{H H}=1\right) \mathrm{HH}$ condition. The HHCP, MC-CP and APHH-CP dynamics were recorded by using the 3.2-mm (E-free)

CPMAS probe with a constant proton spin lock RF field $\omega_{1 I} / 2 \pi=60.4 \mathrm{kHz}$. The ${ }^{1} \mathrm{H}$ and ${ }^{13} \mathrm{C} 90^{\circ}$ pulses applied during the MC-CP cycles (Fig. 1) were fixed at an RFfield strength of $\sim 100 \mathrm{kHz}$ and $\sim 80 \mathrm{kHz}$, respectively. In the APHH-CP experiments at $v_{r}=4 \mathrm{kHz}$, the carbon RF field was swept from 0 to $120.8 \mathrm{kHz}$ through the $\mathrm{HH}$ condition using the tangential time dependence given in Ref. [52] and defined by the shape angle $\phi=\alpha t_{c p} / 2$, where $\alpha$ is the rate of angular change [52]. In principle, the ${ }^{13} \mathrm{C}$ RF field variation must be restricted to $\pm v_{r} / 2$ around the first-order sideband $\mathrm{HH}$ condition as described in Ref. [19]. At $v_{r}=7 \mathrm{kHz}$, the modulation of the field intensity was then limited within $\pm 4 \mathrm{kHz}$ around the $n_{H H}=1$ sideband $\mathrm{HH}$ condition. ${ }^{1} \mathrm{H}$ decoupling during acquisition was obtained by using SPINAL-64 [81]. The CP matching profile $[8 ; 50 ; 82]$ at a given contact time $t_{c p}$ was obtained with the $2.5-\mathrm{mm}$ single-coil CPMAS probe by measuring the integrated peak intensity in the carbon spectrum as a function of the proton RF-field strength $\omega_{1 I}$ while keeping constant the RF amplitude of the carbon channel $\omega_{1 S} / 2 \pi$ at $\sim 100 \mathrm{kHz}$. 160 experiments with variable $\omega_{1 I}$ ranging from $\sim 20$ to $\sim 160 \mathrm{kHz}$ were recorded for each $\mathrm{CP}$ matching profile. The recycle time was $60 \mathrm{~s}$ for ferrocene and $15 \mathrm{~s}$ for $L$-alanine. HHCP and MC-CP dynamics were simulated by using the analytical expressions given in the theoretical section or by integrating numerically the QM master equation, Eq. (13), as described previously [45; 83].

\section{Results and Discussion}


Fig. 8 compares the evolution of the integrated intensity of the ${ }^{13} \mathrm{C}$ NMR spectrum in the HHCP, MC-CP, SAP and APHH-CP experiments for a powder sample of ferrocene under MAS at $v_{r}=4$ and $7 \mathrm{kHz}$. The two-stage character of the carbon polarization build-up [84] is clearly visible in the standard HHCP experiment. Moreover, the absence of large rotational echoes in the HHCP dynamics at short contact times for the centerband matching condition may be attributed to the effects of RF-field inhomogeneity (Fig. 7). More importantly, Fig. 8 shows that MC-CP improves substantially the transfer efficiency over HHCP for contact times higher than $\sim 150 \mu$ s as expected from our theoretical results (Section 2). However, after a maximum reached around the 6th cycle $(N=6)$ of the MC-CP sequence, i.e., at contact times between $\sim 0.5$ and $\sim 1$ ms depending on the duration of the CP steps $t_{c p} / n$, the ${ }^{13} \mathrm{C}$ polarization goes back to values obtained with HHCP (Fig. 8). This decrease in signal intensity is readily attributed to cumulative losses due to $\pi / 2$-pulse imperfections [38] and the finite energy of the $I$-spin bath ( $c f$. Section 2). The fact that the SAP experiment also leads to a significant signal increase over HHCP in the same range of contact times (Fig. 8(a)) means that some spin exchange with the ${ }^{1} \mathrm{H}$ spin bath occurs even for a mixing time as short as $125 \mu$ s ( $c f$. Section 2). As expected, higher signal enhancements are nevertheless obtained when using a long mixing time ( $\tau_{M}=10 \mathrm{~ms}$ ) in MC-CP. Indeed, MC-CP experiments yield an intensity gain factor over HHCP as large as 1.68 and 1.91 at the centerband $\mathrm{HH}$ condition for $v_{r}=4 \mathrm{kHz}$ (Fig. 8(a)) and at the first-order sideband $\mathrm{HH}$ condition for $v_{r}=7 \mathrm{kHz}$ (Fig. 8(b)), respectively. Fig. 8(b) also shows that the polarization transfer critically depends on $t_{c p} / n$ in MC-CP at the centerband matching condition and that the recoupling condition $t_{c p} / n=T_{r} / 2$ provides the highest transfer rate. By contrast, a similar transfer efficiency is observed for $t_{c p} / n=T_{r} / 2$ and $T_{r}$ in MC-CP at the $n_{H H}=$ 1 sideband $\mathrm{HH}$ matching condition (Fig. 8(b)). These results clearly confirm that the type of dipolar recoupling is different in these two cases, as predicted by our theoretical analysis (Section 2). 

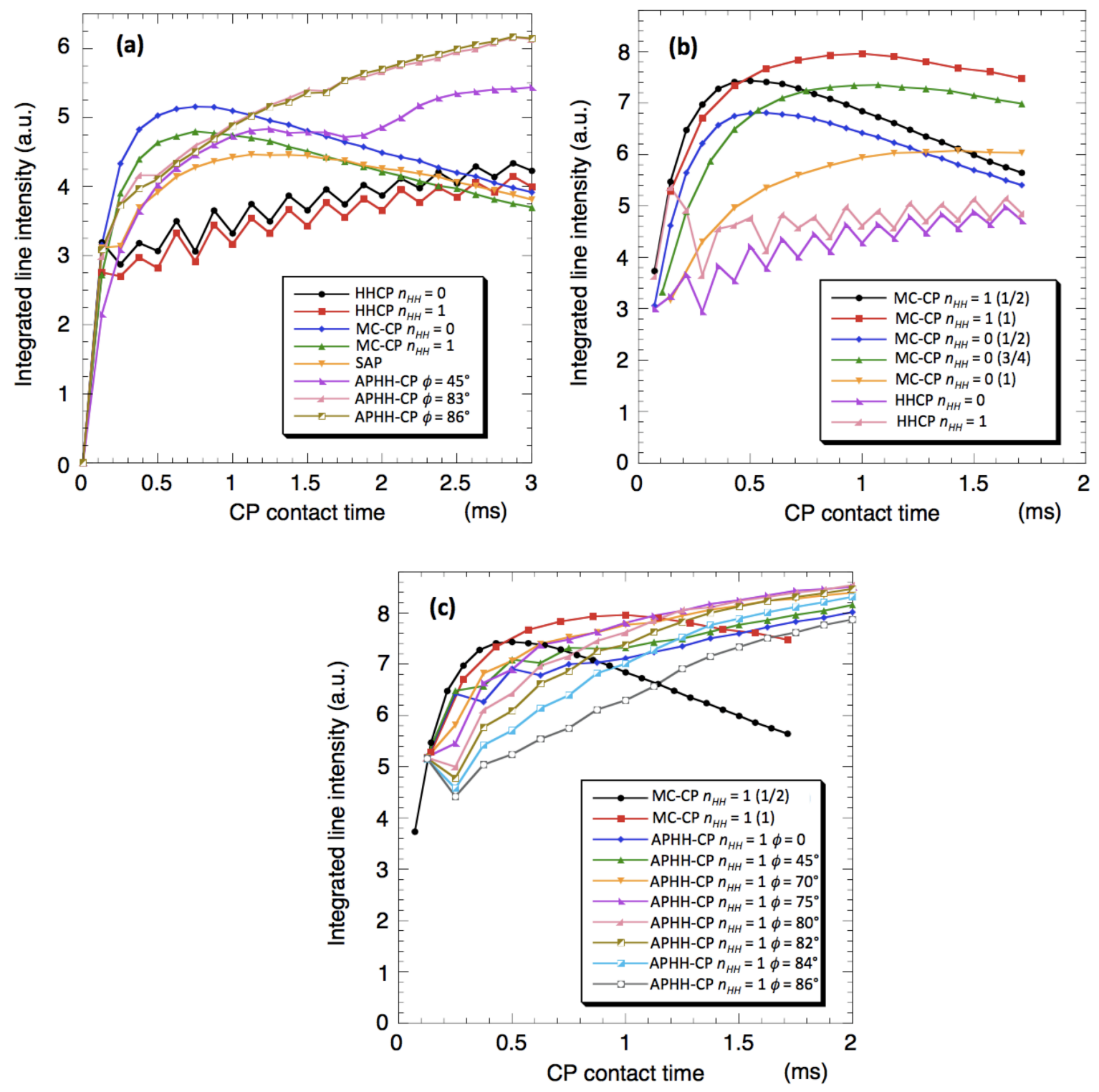

Figure 8 : Integrated intensity of the ${ }^{13} \mathrm{C}$ MAS NMR spectrum of ferrocene as a function of the total contact time $t_{c p}$ for the ${ }^{1} \mathrm{H}-{ }^{13} \mathrm{C}$ HHCP, MC-CP $\left(\tau_{M}=10 \mathrm{~ms}\right)$, SAP and APHH-CP experiments. (a) $v_{r}=4 \mathrm{kHz}\left(T_{r}=250 \mu \mathrm{s}\right): \mathrm{HHCP}\left(n_{H H}=0,1\right)$, SAP $\left(t_{c p} / n=\tau_{M}=T_{r} / 2\right)$, MC-CP $\left(n_{H H}=0,1\right.$ and $\left.t_{c p} / n=T_{r} / 2\right)$ and APHH-CP (full sweep) with shape angle $\phi=45^{\circ}, 83^{\circ}$ and $86^{\circ}$ experiments. (b) $v_{r}=7 \mathrm{kHz}\left(T_{r}=142.86 \mu \mathrm{s}\right)$ : $\operatorname{HHCP}\left(n_{H H}=0,1\right)$ and MC-CP $\left(n_{H H}=0,1\right)$ with $t_{c p} / n=T_{r} / 2(1 / 2), 3 T_{r} / 4(3 / 4)$ or $T_{r}$ (1). (c) $v_{r}=7 \mathrm{kHz}:$ MC-CP $n_{H H}=1$ with $t_{c p} / n=T_{r} / 2(1 / 2)$ or $T_{r}$ (1) and APHH-CP $n_{H H}=1$ with shape angle $\phi=0,45^{\circ}, 70^{\circ}, 75^{\circ}, 80^{\circ}, 82^{\circ}, 84^{\circ}$ and $86^{\circ}$. In order to limit the polarization transfer to the $n_{H H}=1 \mathrm{HH}$ sideband, the APHH-CP sweep width is restricted to $\pm 4 \mathrm{kHz}$. 
Hediger et al. [19] have shown that the CP efficiency can be improved under fast MAS $\left(\omega_{r}>>D_{I S}\right)$ by sweeping the RF amplitude adiabatically through a single sideband $\mathrm{HH}$ matching condition (APHH-CP). However, when the MAS frequency becomes comparable with the heteronuclear dipolar coupling constant the RF amplitude variation must be strictly limited around a given sideband in order to avoid the reverse transfer effects of a sweep through multiple sidebands [19]. Due to this sweep width limitation, an ideal adiabatic behavior cannot be achieved [19]. At a spinning rate of $7 \mathrm{kHz}$, the APHH-CP sweep around the $n_{H H}= \pm 1, \pm 2$ sideband $\mathrm{HH}$ matching condition must typically be restricted so that $\left|\Delta / 2 \pi-n_{H H} v_{r}\right|$ does not exceed $4 \mathrm{kHz}\left(\sim v_{r} / 2\right)[19 ; 20]$. Fig. 8(c) shows the APHH-CP dynamics in ferrocene under MAS at $v_{r}=7 \mathrm{kHz}$ using such a $\pm 4 \mathrm{kHz} \mathrm{RF}$ amplitude variation covering the $n_{H H}=1$ sideband $\mathrm{HH}$ condition for different values of the pulse shape angle $\phi$. It is seen that the polarization transfer efficiency of APHH-CP is critically dependent on $\phi: \phi$ must be less than $\sim 75^{\circ}$ in order to quench completely the transient oscillations and the tangential time dependence is found to be optimum at $\phi \approx 45^{\circ}$ for $t_{c p}<0.3 \mathrm{~ms}$ and at $\phi \approx 70^{\circ}-75^{\circ}$ for longer contact times (Fig. 8(c)). However, MC-CP at the firstorder sideband $\mathrm{HH}$ condition still provides higher signal enhancements than optimized APPH-CP for $t_{c p}<\sim 0.6 \mathrm{~ms}$ if $t_{c p} / n=T_{r} / 2$ and for $t_{c p}<\sim 1 \mathrm{~ms}$ if $t_{c p} / n=$ $T_{r}$ (Fig. 8(c)). Similarly, at a spinning speed of $4 \mathrm{kHz}, \mathrm{MC}-\mathrm{CP}$ at the centerband $\mathrm{HH}$ condition with $t_{c p} / n=T_{r} / 2$ provides a higher signal enhancement that APHH-CP for $t_{c p}<\sim 1 \mathrm{~ms}$ (Fig. 8(a)). Since it is not possible to sweep the RF amplitude through a single $\mathrm{HH}$ condition at this lower spinning frequency the ${ }^{13} \mathrm{C}$ RF field was swept from 0 to $120.8 \mathrm{kHz}$. For such a sweep through multiple sidebands, a significant reduction of the signal has been previously observed [19]. Indeed, Fig. 8(a) shows that the polarization transfer is partially reversed near $t_{c p}=2 \mathrm{~ms}$ when the amplitude sweep approaches a linear variation, $\phi=45^{\circ}$. The tangential time dependence for APHH-CP at $v_{r}=4 \mathrm{kHz}$ is found to be optimum for $\phi \approx 83^{\circ}-86^{\circ}$, as in the case of a static or slowly rotating sample [38]. Due to intensity losses attributed mainly to $\pi / 2$ pulse imperfections, optimized APHH-CP always performs better than $\mathrm{MC}-\mathrm{CP}$ at 
contact times longer than $1 \mathrm{~ms}$ (Fig. 8(a,c)). However, long contact times result in less than maximal signal when the ${ }^{1} \mathrm{H}$ spin-lattice relaxation time in the rotating frame $\left(T_{1 \rho}^{H}\right)$ is short, as in the case of semi-solids such as liquid crystalline materials, polymers, membrane-associated peptides or small proteins $[6 ; 85 ; 86]$. MC-CP is then expected to be particularly useful in mobile samples characterized by a short $T_{1 \rho}$ relaxation time. Note also that $\mathrm{MC}-\mathrm{CP}$ is simpler to implement than $\mathrm{APHH}-\mathrm{CP}$ since there is no need for pulse-shape generation (and/or frequency sweep [18; 22; 23]) and optimization (it is only necessary to match approximately the centerband or a sideband $\mathrm{HH}$ condition).
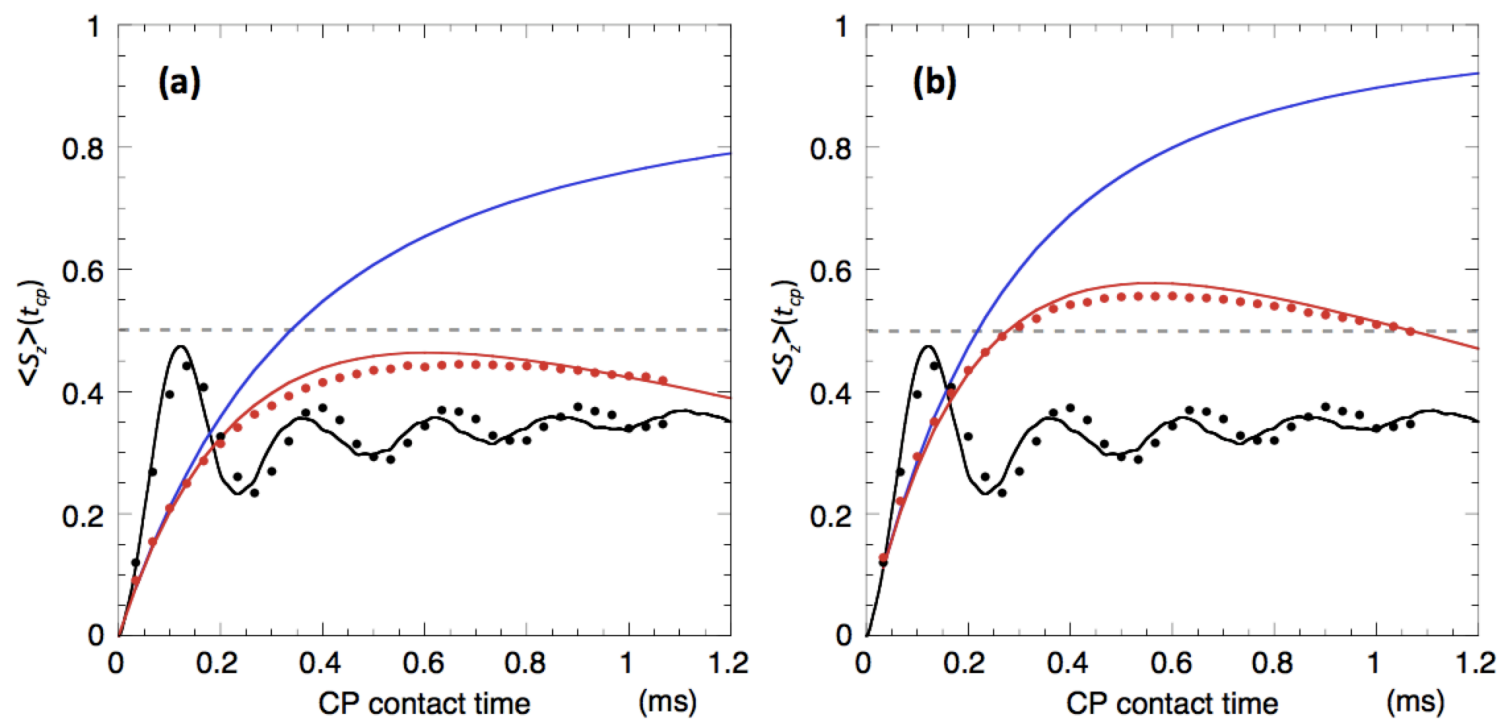

Figure 9: Experimental and calculated ${ }^{1} \mathrm{H}-{ }^{13} \mathrm{C} \mathrm{CP}$ dynamics under MAS at $v_{r}=15$ kHz. Experiments in ferrocene : HHCP with $n_{H H}=1$ (black circles), MC-CP ( $\tau_{M}=10$ ms) with $t_{c p} / n=T_{r} / 2=33.33 \mu$ s and (a) $n_{H H}=0$ or (b) $n_{H H}=1$ (red squares).

Calculations obtained by numerical integration of the QM master equation (Eq. (13)) considering an $\mathrm{HH}$ mismatch distribution with $\sigma_{\Delta} / 2 \pi=3 \mathrm{kHz}$ : HHCP with $\bar{\Delta} / 2 \pi$ $=15 \mathrm{kHz}$ (black line); MC-CP with (a) $\bar{\Delta}=0$ or (b) $\bar{\Delta} / 2 \pi=15 \mathrm{kHz}$ corrected for $\pi / 2$-pulse imperfections by exponential multiplication with an intensity loss of (a) $2 \%$ or (b) $1.9 \%$ per cycle (red line). The blue lines are the corresponding uncorrected MC-CP dynamics calculations. All calculations use an $I^{*}-S$ internuclear distance $r=$ $1.09 \mathrm{~A}$ and the spin diffusion time constants $T_{d p}=1.5 \mathrm{~ms}, T_{d f}=20 \mathrm{~ms}$. 
In summary, the experimental results of Fig. 8 demonstrating that the polarization transfer efficiency of $\mathrm{MC}-\mathrm{CP}$ at short contact times is higher than the one of HHCP confirm our theoretical analysis (Section 2). Furthermore, these data show that MC-CP yields higher signal enhancements at short contact times than optimized APHH-CP when the MAS frequency is comparable with the heteronuclear dipolar coupling, i.e., when APHH-CP through a single sideband matching condition is impossible $\left(v_{r}=4 \mathrm{kHz}\right)$ or difficult to perform $\left(v_{r}=7 \mathrm{kHz}\right)$. It is then of interest to apply MC-CP also at higher spinning speed and to perform a quantitative analysis of the results. Fig. 9 shows the evolution of the carbon magnetization in HHCP at the first-order $\mathrm{HH}$ matching condition $\left(n_{H H}=1\right)$ and $\mathrm{MC}-\mathrm{CP}\left(n_{H H}=0,1\right)$ experiments for a ferrocene powder sample under MAS at a frequency of $15 \mathrm{kHz}$. The two-stage oscillatory behavior in the signal amplitude observed in the HHCP experiment at short contact time agrees well with the calculations of Fig. 2. However, when compared with the MC-CP data, the quasi-equilibrium state polarization reached in the HHCP experiments appears to be much lower than the value predicted by calculation, $\overline{\left\langle S_{z}\right\rangle_{q e}}=1 / 2$ (Fig. 9). As suggested by the calculations of Fig. 6, this observation is attributed to the fact that MC-CP is much less sensitive towards RF mismatch/inhomogeneity between the two channels than HHCP. Indeed, Fig. 9 demonstrates that it is possible to reconcile the HHCP and MC-CP data by considering a $\mathrm{HH}$ mismatch distribution $P(\Delta)$ (Eq. (56)) with $\sigma_{\Delta} / 2 \pi=3 \mathrm{kHz}$. The good agreement between experiments and calculations also shows that SD in the laboratory frame is still fast enough to compensate the local magnetization loss of the directly bound proton with $\tau_{M}=10 \mathrm{~ms}$, even at an MAS speed of $15 \mathrm{kHz}$. For simplicity, $\bar{\Delta} / 2 \pi$ has been fixed to 0 for $n_{H H}=0$ and $15 \mathrm{kHz}$ for $n_{H H}=1$ although slight deviations from these values cannot be excluded [64]. Actually, while this distribution is responsible for the fact that a large part of the sample in a fully filled rotor does not contribute to the HHCP signal, it does not cause any significant loss of intensity for MC-CP. Unfortunately, RF inhomogeneity still has a detrimental effect on the flip angle of the $\pi / 2$ pulses used in MC-CP (Fig. 1). However, despite overall 
losses in intensity estimated from the calculations at $\sim 2 \%$ per cycle (a small fraction of ${ }^{1} \mathrm{H}$ and ${ }^{13} \mathrm{C}$ magnetization is lost after each $\pi / 2$ pulse), the signal intensities at $t_{c p} \approx$ $0.5 \mathrm{~ms}$ provided by MC-CP with $n_{H H}=0$ and $n_{H H}=1$ are higher than the ones obtained with $\operatorname{HHCP}\left(n_{H H}=1\right)$ by a factor $\sim 1.5$ and $\sim 1.9$, respectively (Fig. 9 ). Moreover, the MC-CP signal increases steadily and reaches a large maximum plateau for contact times around $0.5 \mathrm{~ms}$ without the wiggles following the average time course of the HHCP dynamics. The results of Figs 6 and 9 are fully confirmed by the experimental CP matching profiles of Fig. 10 showing that MC-CP broadens significantly the width of the first and second-order $\mathrm{HH}$ conditions and reintroduces efficient $\mathrm{CP}$ at the centerband $\mathrm{HH}$ condition when $t_{c p} / n=T_{r} / 2$. The robustness toward inhomogeneous RF fields demonstrated by Fig. 10 confirms that the MC-CP pulse sequence is little sensitive to instabilities in RF power and/or probe tuning occuring over long periods of signal acquisition. However, by contrast to the results of Fig. 9, note that the intensities of the HHCP $n_{H H}= \pm 1$ sidebands are always comparable or slightly higher than the maxima of MC-CP matching profiles for $t_{c p} / n$ $=T_{r} / 2$ (Fig. 10). This suggests that $\pi / 2$-pulse imperfections attributed mainly to RF inhomogeneity cause a larger loss of polarization than in Fig. 9 although the differential inhomogeneity of the two RF irradiation fields in the single-coil $2.5-\mathrm{mm}$ CPMAS probe is reduced when compared to the $3.2-\mathrm{mm}$ E-free probe, as it must be. Indeed, a significant improvement of the CP efficiency by MC-CP is observed at the $n_{H H}= \pm 1$ and \pm 2 matching conditions when increasing the length of the CP steps (decreasing the number of cycles) : Fig. 10 shows that intensity gains over HHCP $\left(n_{H H}= \pm 1\right)$ by a factor 1.2-1.3 and 1.4-1.5 are obtained with $t_{c p} / n=T_{r}$ and $3 T_{r} / 2$, respectively $\left(t_{c p}=0.2-0.6 \mathrm{~ms}\right)$. 

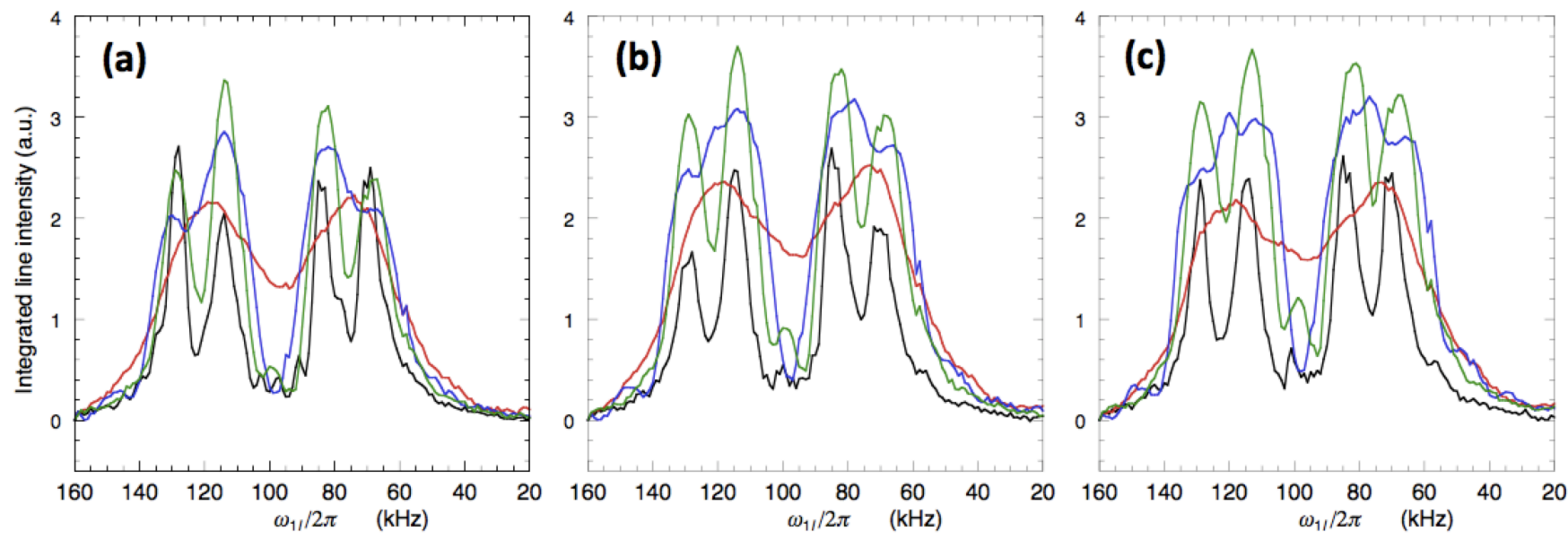

Figure 10 : Integrated line intensity of the carbon resonance in ferrocene as a function of the proton RF-field strength $\omega_{1 I}$ (CP matching profile) at different contact times $t_{c p}$ for HHCP (black line) and MC-CP $\left(\tau_{M}=10 \mathrm{~ms}\right.$ ) with $t_{c p} / n=T_{r} / 2$ (red line), $t_{c p} / n=T_{r}$ (blue line) and $t_{c p} / n=3 T_{r} / 2$ (green line) under MAS at $v_{r}=$ $15 \mathrm{kHz}$. (a) $t_{c p}=0.2 \mathrm{~ms}$; (b) $t_{c p}=0.4 \mathrm{~ms}$; (c) $t_{c p}=0.6 \mathrm{~ms}$. The carbon RF field $\omega_{1 S}$ is equal to $\sim 100 \mathrm{kHz}$.

It is well known that efficient polarization transfer at the original centerband matching condition can also be achieved with amplitude-modulated CP (S-AMCP) and that the width of the matching conditions is efficiently broadened by amplitudeand phase-modulated CP (D-AMCP or APM-CP) [12; 13; 82; 87]. Indeed, the transfer efficiency and broadening of the matching conditions achieved by MC-CP (Fig. 10) are similar to those previously obtained by D-AMCP [78]. However, for ferrocene at $v_{r}=15 \mathrm{kHz}$, we have observed [78] that D-AMCP leads, after a rapid initial increase during the first three rotor cycles, to a decay of the $S$-spin polarization towards the quasi-equilibrium magnetization obtained in HHCP. Hence, due to the oscillating behavior of the D-AMCP dynamics, signal enhancement critically depends on the contact time. Moreover, by contrast to D-AMCP, the MC-CP pulse scheme is not affected by the destructive effect of DQ CP [78] but suffers from $\pi / 2$ pulse imperfections. Fig. 3(a) of Ref. [20] shows that optimized AMAP-CP at $v_{r}=$ $17 \mathrm{kHz}$ in ferrocene yields a higher signal intensity than $\operatorname{HHCP}\left(n_{H H}= \pm 1\right)$ by a factor $\sim 1.5$ for $t_{c p} \approx 0.4 \mathrm{~ms}$. Fig. 10 shows that such a sensitivity enhancement is only 
slightly higher than the one obtained by MC-CP with $t_{c p} / n=3 T_{r} / 2$. APHH-CP [19] or, preferably, AMAP-CP [20] is nevertheless expected to give better results than MC-CP at longer contact times due to the $\pi / 2$-pulse imperfections (Fig. 8).
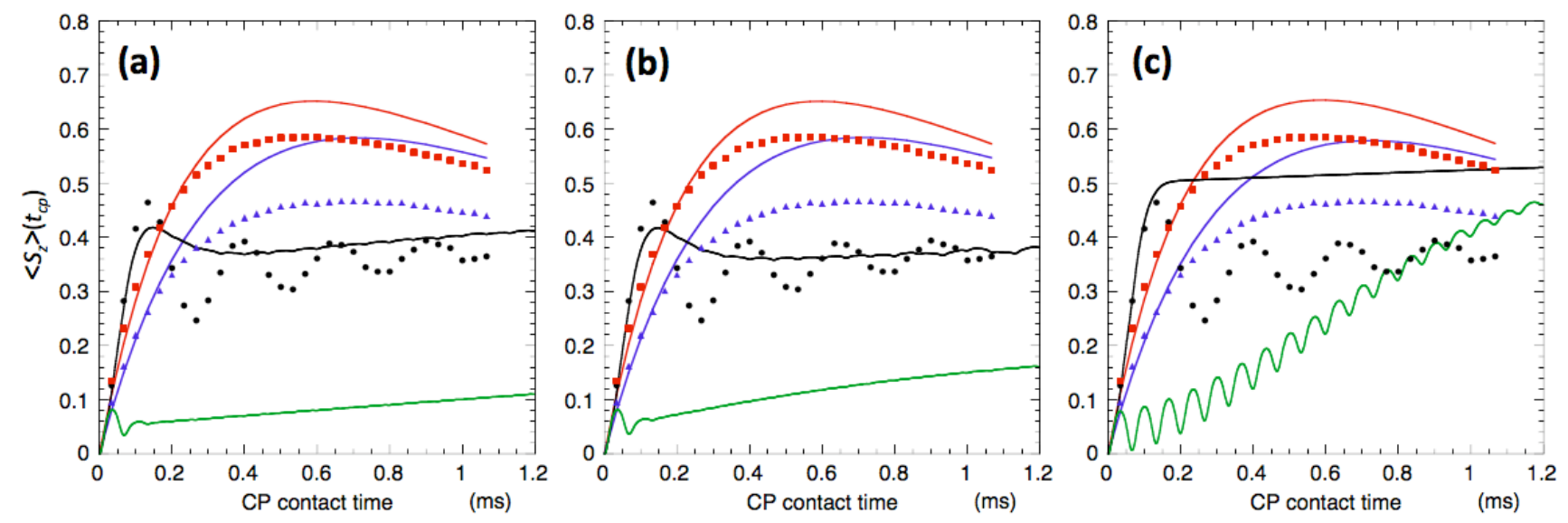

Figure 11 : Experimental and calculated ${ }^{1} \mathrm{H}-{ }^{13} \mathrm{C} \mathrm{CP}$ dynamics under MAS at $v_{r}=15$ $\mathrm{kHz}$. Experiments in ferrocene : HHCP with $n_{H H}=1$ (black circles), MC-CP ( $\tau_{M}=10$ ms and $t_{c p} / n=T_{r} / 2$ ) with $n_{H H}=1$ (red squares) and $n_{H H}=0$ (blue triangles). Calculations obtained by a (two-step) memory function approach in the AW approximation : HHCP with $\bar{\Delta} / 2 \pi$ (or $\Delta / 2 \pi)=15 \mathrm{kHz}$ (black line), MC-CP with $\bar{\Delta} / 2 \pi$ (or $\Delta / 2 \pi)=15 \mathrm{kHz}$ (red line), MC-CP with $\bar{\Delta}$ (or $\Delta$ ) $=0$ (blue line), HHCP with $\bar{\Delta}$ (or $\Delta$ ) $=0$ (green line). (a) Fast fluctuation (FF) approximation with $\sigma_{\Delta} / 2 \pi$ $=3 \mathrm{kHz}$; (b) two-step memory function (TS-MF) approach with $\sigma_{\Delta} / 2 \pi=3 \mathrm{kHz}$; (c) TS-MF approach with no HH mismatch distribution $\left(\sigma_{\Delta}=0\right) . T_{d p}=10 \mathrm{~ms}$ in the FF approximation ; $1 / \sqrt{N_{2}^{I I}}=0.1 \mathrm{~ms}$ and $\tau_{x}=4.8 \mathrm{~ms}$ in the TS-MF approach. All calculations use $1 / \sqrt{M_{2}^{I S}}=30.67 \mu \mathrm{s}, T_{d f}=20 \mathrm{~ms}$. The MC-CP dynamics calculations are corrected for $\pi / 2$-pulse imperfections by exponential multiplication with an intensity loss of $1.7 \%$ per cycle.

The data of Fig. 9 clearly demonstrate that the HHCP transfer efficiency is strongly reduced by RF-field inhomogeneity in our 3.2-mm E-free probe. However, this analysis required rather tedious computations involving exact numerical integration of the QM master equation (Eq. (13)). Indeed, the independent sideband approximation is not valid when $v_{r}=15 \mathrm{kHz}$ ( $c f$. Fig. 5) and mismatch of the $\mathrm{HH}$ 
condition is not accounted for by the MKBE-MAS solutions ( $c f$. Section 2). Note however that Eq. (32) can be employed to successfully simulate the MC-CP data of Fig. 9 at the centerband $\mathrm{HH}$ matching condition because MC-CP is little sensitive to HH mismatch (Fig. 6). In Section 2, we have derived simple NS analytical solutions for HHCP and MC-CP dynamics that are valid for arbitrary values of the applied RF fields and spinning frequency by using a combination of the MF approach and the AW approximation. Therefore, it is of interest to analyze the data of Fig. 9 with these analytical expressions both in the FF approximation and by the TS-MF approach. The corresponding simulations are displayed in Fig. 11. Although the Gaussian frequency distribution model does not account for the transient oscillations observed in HHCP at the first-order sideband $\mathrm{HH}$ matching condition ( $c f$. Fig. 5) it is seen that a $\mathrm{HH}$ mismatch distribution must be considered in order to obtain a good agreement with the quasi-equilibrium state polarization reached at contact times longer than $\sim 0.5 \mathrm{~ms}$ both in the FF approximation (Fig. 11(a)) and with the TS-MF approach (Fig. 11(b)). Indeed, as for the exact powder numerical calculations of Fig. 9, the quasiequilibrium state polarization is found to be largely overestimated when the effects of RF-field inhomogeneity on HHCP are neglected (Fig. 11(c)). As expected, the MCCP data of Fig. 11 are well fitted by the AW model at short contact times ( $c f$. Fig. 5) and are not affected by a $\mathrm{HH}$ mismatch distribution with $\sigma_{\Delta} / 2 \pi=3 \mathrm{kHz}$. Although the TS-MF approach is used in Fig. 11(c) a similar result is obtained in the FF approximation for the HHCP dynamics with $n_{H H}=1$ (data not shown). This is not surprising recalling that HHCP dynamics at the first-order $\mathrm{HH}$ matching condition is little affected by memory effects of the $I$-spin bath (Fig. 7). As already mentioned, the strength of the TS-MF approach is to better accounts for the polarization transfer at the centerband $\mathrm{HH}$ matching condition (Fig. 7). Hence, calculations of the HHCP dynamics for $\bar{\Delta}$ or $\Delta=0$ are also displayed in Fig. 11. The increase in polarization transfer efficiency due to the modulation of the $I^{*}-I$ SE interactions by sample spinning is clearly visible, especially in the absence of $\mathrm{HH}$ mismatch distribution (Fig. 11(c)). Our previous experimental HHCP data at $v_{r}=15 \mathrm{kHz}$ showing that 
some polarization transfer also occurs at the centerband $\left(n_{H H}=0\right)$ for $t_{c p}>\sim 0.6 \mathrm{~ms}$ [78] support the presence of secondary recoupling effects in ferrocene. This means that $C_{x}(t)$ deviates slightly from an exponential decay at this spinning speed ( $c f$. Section 2). This result must be associated with the inhomogeneous character of the ${ }^{1} \mathrm{H}-{ }^{1} \mathrm{H}$ interactions in ferrocene leading to the presence of resolved rotational sidebands in the SD spectrum [59]. Indeed, the homogeneous proton SD time constant $T_{S}$ measured by the ${ }^{13} \mathrm{C}$-detected SD experiments developed by Zhang et al [59] increases linearly with the MAS frequency even at low spinning rates $\left(v_{r} \sim 1\right.$ $\mathrm{kHz}$ ). The slowing down of SD by MAS in ferrocene also leads to a partially inhomogeneous character of the proton spectrum with well-separated spinning sidebands [88]. We have remarked that the experimental ${ }^{1} \mathrm{H}$ MAS spectrum of ferrocene at $v_{r}=5 \mathrm{kHz}$ published in Fig. 4 of Ref. [88] is well fitted by the AW theory $[73 ; 89]$ using the ${ }^{1} \mathrm{H}$ second moment measured by Kubo et al $[90], 1 / \sqrt{M_{2}^{I I}}=$ $29.5 \mu \mathrm{s}$, and a bath-correlation time associated with $\mathrm{SD}, \tau_{B}=0.4 \mathrm{~ms}$. This latter value is found to be in good agreement with the SD decay in the laboratory frame measured at $v_{r}=4 \mathrm{kHz}$ by Zhang et al. [59]. Note also that the AW stochastic theory of the NMR lineshape developed by Clough and Gray [89] leads to a linear dependence of the SD time constant on the MAS frequency when $\omega_{r}^{2} \gg M_{2}^{I I}$ in good agreement with local proton SD measurements [59;60]. This suggests that a good estimation of the correlation time of the $I$-spin bath $\tau_{x}$ is given by the experimental SD time constant in the rotating frame $T_{S}$ corresponding to the homogeneous part of the dipolar interaction. $T_{S}$ is plotted for MAS frequencies in the range from 1 to $8 \mathrm{kHz}$ in Fig. 7 of Ref. [59]. In fact, the value of $\tau_{x}=4.8 \mathrm{~ms}$ used for the calculations of Fig. 11 has been fixed to the SD time constant $T_{S}$ obtained by extrapolating the data of Zhang et al. [59] to a MAS rate of $15 \mathrm{kHz}$. The same analysis of the CP dynamics is performed in Fig. 12 for $v_{r}=7 \mathrm{kHz}$ with $\tau_{x}=T_{S}=$ $2.3 \mathrm{~ms}$ [59]. A good agreement with the MC-CP experimental data is again obtained at short contact times when considering a $\mathrm{HH}$ mismatch distribution with $\sigma_{\Delta} / 2 \pi=3$ kHz both in the FF approximation (Fig. 12(a,b)) and with the TS-MF approach (Fig. 
12(c,d)). However, the TS-MF approach provides a better agreement with the HHCP experimental data during the second stage of the polarization transfer. Indeed, memory effects of the $I$-spin bath cannot be neglected because $\tau_{x}=2.3 \mathrm{~ms}$ is still quite long at $v_{r}=7 \mathrm{kHz}$ [59]. The remaining discrepancy with the HHCP experiments (Fig. 12(c,d)) may be attributed to the fact that only the Ising term of the SE interaction is considered in the TS-MF approach (the secular approximation is used for the XY term). Note that the small rotational echoes already noticed in Fig. 7 are indeed present in the HHCP experiments. Fig. 12(e,f) shows that RF-field inhomogeneity has an important effect on $\mathrm{HHCP}$ and $\mathrm{MC}-\mathrm{CP}$ at the centerband $\mathrm{HH}$ matching condition for $t_{\mathrm{cp}} / n=3 T_{r} / 4$ and $T_{r}$. As in Fig. 11(c), the quasi-equilibrium state polarization reached in $\mathrm{HHCP}\left(n_{H H}=0,1\right)$ is overestimated by the calculations with no $\mathrm{HH}$ mismatch distribution. On the other hand, the MC-CP $\left(n_{H H}=0\right)$ polarization transfer efficiency is underestimated by the simulations of Fig. 12(e,f) when $t_{\mathrm{cp}} / n=3 T_{r} / 4$ and $T_{r}$. This result illustrates the fact that MC-CP becomes more dependent on mismatch of the $\mathrm{HH}$ condition when increasing $t_{\mathrm{cp}} / n$. Fig. 13 shows that the TS-MF approach in the AW approximation also gives a good agreement with experiment at $v_{r}=4 \mathrm{kHz}$. Finally, the inverse of the square root of the ${ }^{1} \mathrm{H}$ second moment $1 / \sqrt{M_{2}^{I I}}=29.5 \mu$ s may be compared with the value of $1 / \sqrt{N_{2}^{I I}}=0.1 \mathrm{~ms}$ used to fit all the HHCP and MC-CP experiments at $v_{r}=4,7$ and $15 \mathrm{kHz}$ (Figs 11, 12 and 13). The experimental ratio $\sqrt{N_{2}^{I I} / M_{2}^{I I}} \approx 0.295$ is found to be close to the expected value of $1 / 3$ ( $c f$. Section 2). 

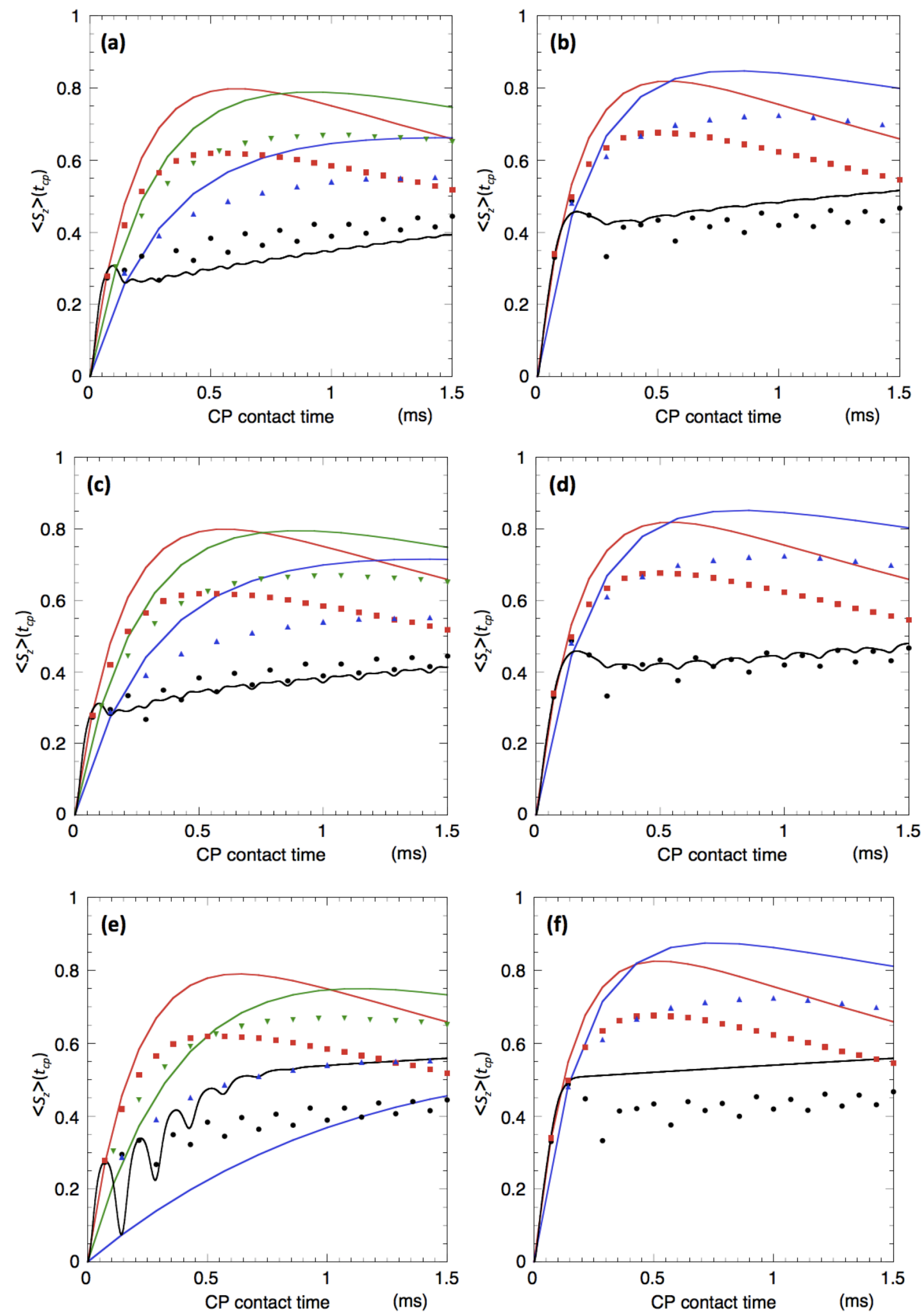

Figure 12: Experimental and calculated ${ }^{1} \mathrm{H}-{ }^{13} \mathrm{C} \mathrm{CP}$ dynamics under MAS at $v_{r}=7$ $\mathrm{kHz}$. Experiments in ferrocene with (a,c,e) $n_{H H}=0$ and (b,d,f) $n_{H H}=1:$ HHCP (black circles) ; $\mathrm{MC}-\mathrm{CP}\left(\tau_{M}=10 \mathrm{~ms}\right)$ with $t_{c p} / n=T_{r} / 2$ (red squares), $t_{c p} / n=3 T_{r} / 4$ (green up triangles) and $t_{c p} / n=T_{r}$ (blue down triangles). Calculations with $(\mathrm{a}, \mathrm{c}) \bar{\Delta}=$ 
0 , (e) $\Delta=0$, (b,d) $\bar{\Delta} / 2 \pi=7 \mathrm{kHz}$ and (f) $\Delta / 2 \pi=7 \mathrm{kHz}$ : HHCP (black line); MCCP with $t_{c p} / n=T_{r} / 2$ (red line), $t_{c p} / n=3 T_{r} / 4$ (green line); HHCP with $t_{c p} / n=T_{r}$ (blue line). (a,b) FF approximation with $\sigma_{\Delta} / 2 \pi=3 \mathrm{kHz}$; (c,d) TS-MF approach with $\sigma_{\Delta} / 2 \pi=3 \mathrm{kHz}$; (e,f) TS-MF approach with no $\mathrm{HH}$ mismatch distribution ( $\sigma_{\Delta}$ =0). $T_{d p}=10 \mathrm{~ms}$ in the FF approximation ; $1 / \sqrt{N_{2}^{I I}}=0.1 \mathrm{~ms}$ and $\tau_{x}=2.3 \mathrm{~ms}$ in the TS-MF approach. All calculations use $1 / \sqrt{M_{2}^{I S}}=30.67 \mu$ s and $T_{d f}=12 \mathrm{~ms}$. The MC-CP dynamics calculations are corrected for $\pi / 2$-pulse imperfections by exponential multiplication with an intensity loss of $2 \%$ per cycle.
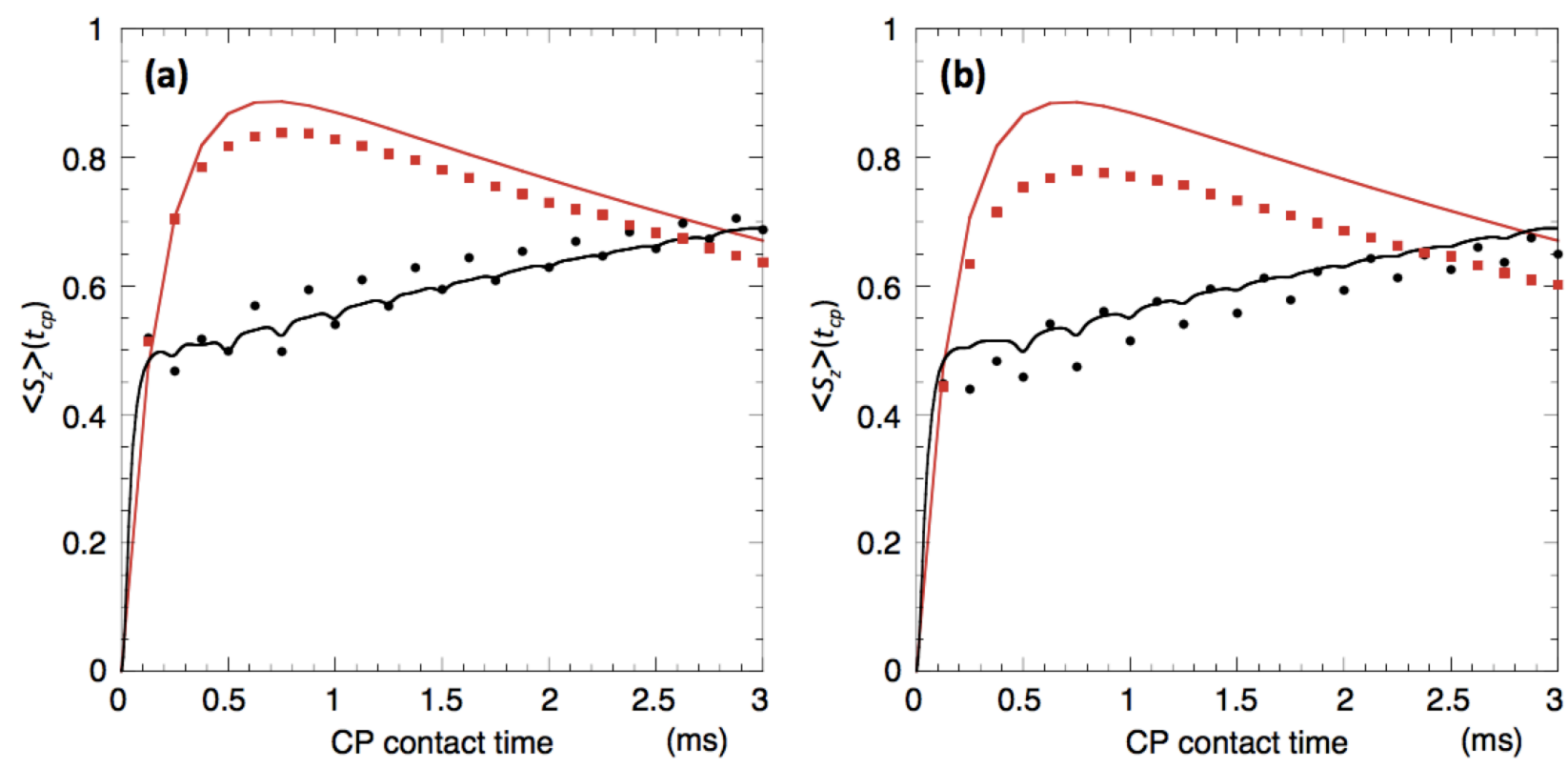

Figure 13 : Experimental and calculated ${ }^{1} \mathrm{H}-{ }^{13} \mathrm{C} \mathrm{CP}$ dynamics under MAS at $v_{r}=4$ $\mathrm{kHz}$. Experiments in ferrocene with (a) $n_{H H}=0$ and (b) $n_{H H}=1: \mathrm{HHCP}$ (black circles); MC-CP with $\tau_{M}=10 \mathrm{~ms}$ and $t_{c p} / n=T_{r} / 2$ (red squares). Calculations with (a) $\bar{\Delta}=0$ and (b) $\bar{\Delta} / 2 \pi=4 \mathrm{kHz}$ : HHCP (black line); MC-CP with $t_{c p} / n=T_{r} / 2$ (red line). (a,b) TS-MF approach with $\sigma_{\Delta} / 2 \pi=3 \mathrm{kHz}, 1 / \sqrt{N_{2}^{I I}}=0.1 \mathrm{~ms}$ and $\tau_{x}=$ $1.5 \mathrm{~ms}$. All calculations use $1 / \sqrt{M_{2}^{I S}}=30.67 \mu \mathrm{s}$ and $T_{d f}=6 \mathrm{~ms}$. The MC-CP dynamics calculations are corrected for $\pi / 2$-pulse imperfections by exponential multiplication with an intensity loss of $1.7 \%$ per cycle. 
We have clearly demonstrated that MC-CP experiments improve the polarization transfer in ferrocene. Ferrocene may nevertheless be considered as an untypical sample due to the uniaxial rotational molecular motion of the rings giving an inhomogeneous character to the intramolecular dipolar interactions [59]. Moreover, $T_{1 \rho}$ and $T_{1}$ relaxation has no significant effect on the HHCP and MC-CP data presented above since the conditions $t_{c p} \ll T_{1 \rho}^{I}, T_{1 \rho}^{S}$ and $\tau_{M} \ll<T_{1}^{I}, T_{1}^{S}$ are always verified. Therefore, we have carried out the same experiments on polycrystalline $L$-alanine which exhibits no significant molecular motion at the $\mathrm{C}_{\alpha}$ carbon ( $\mathrm{CH}$ group) and is characterized by a rather short ${ }^{1} \mathrm{H}$ spin-lattice relaxation time in the rotating frame $\left(T_{1 \rho}^{H} \approx 2 \mathrm{~ms}\right)[41 ; 91 ; 92]$. On the other hand, the spinlattice relaxation in the rotating frame of the rare ${ }^{13} \mathrm{C}$ spin can be neglected because it is much weaker ( $T_{1 \rho}^{C} \approx 20 \mathrm{~ms}$ for the $\mathrm{C}_{\alpha}$ carbon) [41; 93]. Fig. 14 compares the HHCP and MC-CP dynamics of the $\mathrm{C}_{\alpha}$ signal of $L$-alanine at $v_{r}=8 \mathrm{kHz}$ for $\tau_{M}$ values ranging from $1 \mathrm{~ms}$ to $10 \mathrm{~s}\left(t_{c p} / n=T_{r} / 2=62.5 \mu \mathrm{s}\right)$. Because of fast $T_{1 \rho}^{H}$ relaxation, the maximum of the HHCP buildup curve is located at $t_{c p} \approx 70 \mu$ s (top of the first dipolar oscillation). In the second stage, the ${ }^{13} \mathrm{C}$ spin magnetization decreases together with the ${ }^{1} \mathrm{H}$ spin magnetization at a rate $1 / T_{1 \rho}^{H}$ in good agreement with previous measurements [92]. The ${ }^{1} \mathrm{H}$ spin-lattice relaxation time in the laboratory frame $T_{1}^{H}$ was determined to be $\sim 3 \mathrm{~s}$. Fig. 14 shows that the ${ }^{13} \mathrm{C}$ polarization decays after a maximum reached already at the first cycle $(N=1)$ of the MC-CP sequence when $\tau_{M}$ is much smaller than $T_{1}^{H}\left(\tau_{M}=1-100 \mathrm{~ms}\right)$. Considering the reported above results, this fast decay cannot be attributed solely to $T_{1 \rho}^{H}$ relaxation and $\pi / 2$ pulse imperfections. As a consequence, $\mathrm{MC}-\mathrm{CP}$ provides an intensity gain over the maximum of the HHCP buildup curve $\left(t_{c p}=62.5 \mu \mathrm{s}\right)$ by a factor $\sim 1.15$ using a single re-equilibration with the proton bath $(N=1)$. Fig. 14 shows that the signal decay disappears when $\tau_{M}$ is comparable to or larger than $T_{1}^{H}\left(\tau_{M}=1-10 \mathrm{~s}\right)$. Indeed, the MC-CP technique then alleviates most of the magnetization loss due to $T_{1 \rho}^{H}$ relaxation through ${ }^{1} \mathrm{H}$ repolarization by $T_{1}^{H}$ relaxation during $\tau_{M}$ [27; 34]. In this range of mixing time, the ${ }^{13} \mathrm{C}$ polarization is essentially independent of the contact time with a 
maximum of only a factor $\sim 1.23$ over $\mathrm{HHCP}\left(t_{c p}=62.5 \mu \mathrm{s}\right)$ for $\tau_{M}=3 \mathrm{~s}$ and $N=3$ (Fig. 14). Such a sensitivity enhancement is too low taking into account the increase of time ( $9 \mathrm{~s}$ ) per scan due to the mixings steps (the recycle time is $15 \mathrm{~s}$ ). This poor performance of MC-CP in $L$-alanine is readily attributed to ${ }^{13} \mathrm{C}$ spin-lattice relaxation in the laboratory frame during $\tau_{M}$. Indeed, the decrease of ${ }^{13} \mathrm{C}$ magnetization observed when increasing further the mixing time to $10 \mathrm{~s}$ (Fig. 14) can only be explained by a non-negligible effect of $T_{1}^{C}$ relaxation [27; 34]. The fact that $T_{1}^{C}$ is not much longer than $T_{1}^{H}$ in $L$-alanine is also consistent with the relaxation time measurements performed by Akasaka et al [93] showing that $T_{1}^{C}=4.0 \mathrm{~s}$ for the $\mathrm{C}_{\alpha}$ carbon at a frequency of $50.3 \mathrm{MHz}$ for ${ }^{13} \mathrm{C}$ resonance. In conclusion, when relying on spin-lattice relaxation mechanisms for ${ }^{1} \mathrm{H}$ repolarization $\left(\tau_{M}>\sim T_{1}^{H}\right)$, the MC-CP technique must strictly be restricted to samples with $T_{1}^{C} \gg T_{1}^{H}$.

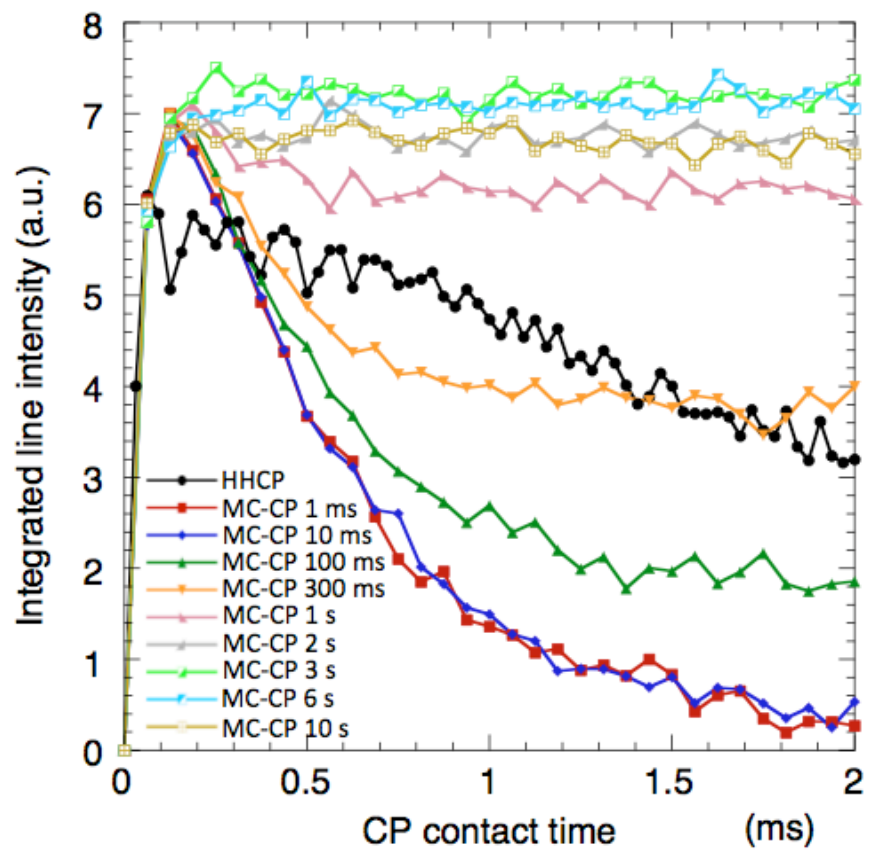

Figure 14 : Integrated intensity of the $\mathrm{C}_{\alpha}$ carbon of $L$-alanine as a function of the total contact time $t_{c p}$ at $v_{r}=8 \mathrm{kHz}\left(n_{H H}=1\right)$ for the HHCP and MC-CP experiments with $\tau_{M}$ ranging from $1 \mathrm{~ms}$ to $10 \mathrm{~s}\left(t_{c p} / n=T_{r} / 2=62.5 \mu \mathrm{s}\right)$. 


\section{Conclusion}

Several analytical expressions to describe HHCP and MC-CP dynamics under MAS have been presented using the $I-I^{*}-S$ model. Exact powder secular solutions have shown that the polarization transfer efficiency can be improved by MC-CP after as few as 4-6 contacts with an effective suppression of transient oscillations. Moreover, using the memory function (MF) approach and the Anderson-Weiss (Gaussian powder) approximation, we have obtained non-secular solutions in the fast fluctuation approximation valid for arbitrary values of the MAS rate and the applied RF fields that give a better agreement with exact numerical integration of the quantum mechanical master equation than the exact powder secular solutions. More importantly, our analysis shows that $\mathrm{MC}-\mathrm{CP}$ is generally much more robust to $\mathrm{RF}$ mismatch/inhomogeneity than HHCP. Memory effects of the $I$-spin bath have also been considered by relaxing the fast fluctuation approximation using a two-step MF (TS-MF) approach. These theoretical results are clearly confirmed by experiments applied to a powder sample of ferrocene. Indeed, we have observed that MC-CP yields a significant gain (of a factor 1.2-1.9) in signal at short contact times as compared to standard HHCP and the widely used APHH-CP technique when the adiabatic transfer is not possible or well optimized. In addition, experimental MC-CP $\mathrm{HH}$ profiles show a significant broadening of the first and second-order sideband matching conditions as well as a reintroduction of efficient polarization transfer at the centerband matching condition. Finally, it is shown that HHCP and MC-CP experimental data at several MAS frequencies are well described by simple analytical solutions provided that RF inhomogeneity and memory effects of the $I$-spin bath (TSMF approach) are considered. It is concluded that the MC-CP technique, which may be further improved by reducing $\pi / 2$-pulse imperfections using, e.g., composite pulses [94], is a simple and robust CPMAS method requiring no pulse-shape optimization. Recent studies $[34 ; 95]$ demonstrate that MC-CP is also applicable to 
disordered systems characterized by a short spin-lattice relaxation time of the $I$ spins in the rotating frame $\left(T_{1 \rho}^{I}\right)$. However, our results obtained in $L$-alanine underline that an important boundary condition for the successful use of the experiment is that the spin-lattice relaxation time of the $S$ spins in the laboratory frame $\left(T_{1}^{S}\right)$ be much longer than $T_{1}^{I}$.

\section{Acknowledgements :}

The authors are grateful to the French ministry of research, the CNRS, the University of Strasbourg and the international center for Frontier Research in Chemistry (icFRC) for their help in establishing and maintaining the solid-state NMR platform. This research did not receive any specific grant from funding agencies in the public, commercial, or not-for-profit sectors. 


\section{References}

[1] A. Pines, M.G. Gibby, and J.S. Waugh, Proton-Enhanced Nuclear Induction Spectroscopy. A Method for High Resolution NMR of Dilute Spins in Solids. J. Chem. Phys. 56 (1972) 1776.

[2] A. Pines, M.G. Gibby, and J.S. Waugh, Proton-enhanced NMR of dilute spins in solids. J. Chem. Phys. 59 (1973) 569.

[3] J. Schaefer, and E.0. Stejskal, Carbon-13 Nuclear magnetic resonance of polymers spinning at the magic angle. J. Am. Chem. Soc. 98 (1976) 1031-1032.

[4] M. Mehring, Principles of High Resolution NMR in Solids, Springer-Verlag, Berlin, 1983.

[5] E.O. Stejskal, and J.D. Memory, High Resolution NMR in the Solid State: Fundamentals of CP/MAS, Oxford Univ. Press, New York, 1994.

[6] W. Kolodziejski, and J. Klinowski, Kinetics of Cross-Polarization in Solid-State NMR: A Guide for Chemists. Chem. Rev. 102 (2002) 613.

[7] S.R. Hartmann, and E.L. Hahn, Nuclear Double Resonance in the Rotating Frame. Phys. Rev. 128 (1962) 2042.

[8] E.0. Stejskal, J. Schaefer, and J.S. Waugh, Magic-angle spinning and polarization transfer in proton-enhanced NMR. J. Magn. Reson. 28 (1977) 105-112.

[9] B.H. Meier, Cross polarization under fast magic angle spinning: thermodynamical considerations. Chem. Phys. Lett. 188 (1992) 201-207.

[10] S. Ding, C.A. McDowell, and C. Ye, Cross polarization at the slow-correlation limit. J. Magn. Reson. Ser. A 109 (1994) 1-5.

[11] S. Ding, C.A. McDowell, and C. Ye, Dynamic theory of cross-polarization in solids under high-speed magic-angle spinning. J. Magn. Reson. Ser. A 109 (1994) 6-13.

[12] X. Wu, and K. Zilm, Cross polarization with high speed magic-angle spinning. J. Magn. Reson. Ser. A 104 (1993) 154-165.

[13] S. Hediger, B.H. Meier, and R.R. Ernst, Cross polarization under fast magic angle sample spinning using amplitude-modulated spin-lock sequences. Chem. Phys. Lett. 213 (1993) 627-635.

[14] R. Pratima, and K.V. Ramanathan, Synchronous accumulation of polarisation. A new approach to cross polarisation under fast magic angle spinning. Chem. Phys. Lett. 221 (1994) 322-326.

[15] O.B. Peersen, X. Wu, I. Kustanovich, and S.O. Smith, Variable-Amplitude CrossPolarization MAS NMR. J. Magn. Reson. Ser. A 104 (1993) 334.

[16] G. Metz, X. Wu, and S.O. Smith, Ramped-Amplitude Cross polarization in Magic-AngleSpinning NMR. J. Magn. Reson. Ser. A 110 (1994) 219.

[17] A.C. Kolbert, and S.L. Gann, Variable-effective-field cross polarization. An approach to broadband Hartmann-Hahn matching in MAS NMR. Chem. Phys. Lett. 224 (1994) 86.

[18] A.C. Kolbert, and A. Bielecki, Broadband Hartmann-Hahn matching in magic-angle spinning NMR via adiabatic frequency sweep. J. Magn. Reson. Ser. A 116 (1995) 29-35.

[19] S. Hediger, B.H. Meier, and R.R. Ernst, Adiabatic passage Hartmann-Hahn cross polarization in NMR under magic angle sample spinning. Chem. Phys. Lett. 240 (1995) 449-456.

[20] S. Hediger, P. Signer, M. Tomaselli, R.R. Ernst, and B.H. Meier, A combination of slow and fast RF field modulation for improved cross polarization in solid-state MAS NMR. J. Magn. Reson. 125 (1997) 291-301.

[21] R. Fu, P. Pelupessy, and G. Bodenhausen, Frequency-modulated cross-polarization for fast magic angle spinning NMR at high fields: relaxing the Hartmann-Hahn condition. Chem. Phys. Lett. 264 (1997) 63. 
[22] W.K. Peng, K. Takeda, and M. Kitagawa, A new technique for cross polarization in solidstate NMR compatible with high spinning frequencies and high magnetic fields. Chem. Phys. Lett. 417 (2006) 58.

[23] W.K. Peng, and K. Takeda, Efficient cross polarization with simultaneous adiabatic frequency sweep on the source and target channels. J. Magn. Reson. 188 (2007) 267.

[24] S. Jain, M. Bjerring, and N.C. Nielsen, Efficient and robust heteronuclear crosspolarization for high speed-spinning biological solid-state NMR spectroscopy. J. Phys. Chem. Lett. 3 (2012) 703-708.

[25] A.B. Nielsen, S. Jain, M. Ernst, B.H. Meier, and N.C. Nielsen, Adiabatic rotor-echo-shortpulse-irradiation mediated cross-polarization. J. Magn. Reson. 237 (2013) 147-151.

[26] L.B. Alemany, D.M. Grant, R.J. Pugmire, T.D. Alger, and K.W. Zilm, Cross polarization and magic angle sample spinning NMR spectra of model organic compounds. 1. Highly protonated molecules. J. Am. Chem. Soc. 105 (1983) 2133-2141.

[27] N. Zumbulyadis, and J.M. O'Reilly, Cross polarization in systems with heterogeneous proton distribution. J; Magn. Reson. 82 (1989) 613-618.

[28] G. Jeschke, and G. Grossmann, Spinning-Sideband-Pattern Deviations in CrossPolarization MAS NMR Spectra. J. Magn. Reson. Ser. A 103 (1993) 323.

[29] G. Metz, M. Ziliox, and S.O. Smith, Towards quantitative CP-MAS NMR. Solid State Nucl. Magn. Reson. 7 (1996) 155-160.

[30] R. Fu, J. Hu, and T.A. Cross, Towards quantitative measurements in solid-state CPMAS NMR: A Lee-Goldburg frequency modulated cross-polarization scheme. J. Magn. Reson. 168 (2004) 8-17.

[31] G. Hou, F. Deng, S. Ding, R. Fu, J. Yang, and C. Ye, Quantitative cross-polarization NMR spectroscopy in uniformly 13C-labeled solids. Chem. Phys. Lett. 421 (2006) 356-360.

[32] G. Hou, F. Deng, C. Ye, and S. Ding, Towards uniform enhancement in solid-state cross polarization magic angle spinning NMR: A scheme incorporating cross polarization with rotational resonance. J. Chem. Phys. 124 (2006) 234512.

[33] K. Takeda, Y. Noda, K. Takegoshi, O. Lafon, J. Trebosc, and J.P. Amoureux, Quantitative cross-polarization at magic-angle spinning frequency of about $20 \mathrm{kHz}$. J. Magn. Reson. 214 (2012) 340-345.

[34] R.L. Johnson, and K. Schmidt-Rohr, Quantitative solid-state 13C NMR with signal enhancement by multiple cross polarization. J. Magn. Reson. 239 (2014) 44-49.

[35] B.C. Gerstein, and C.R. Dybowski, Transient Techniques in NMR of Solids: An Introduction to Theory and Practice, Academic Press, San Diego, 1985.

[36] W. Tang, and A.A. Nevzorov, Repetitive cross-polarization contacts via equilibration-reequilibration of the proton bath: Sensitivity enhancement for NMR of membrane proteins reconstituted in magnetically aligned bicelles. J. Magn. Reson. 212 (2011) 245.

[37] S.N. Koroloff, and A.A. Nevzorov, Optimization of cross-polarization at low radiofrequency fields for sensitivity enhancement in solid-state NMR of membrane proteins reconstituted in magnetically aligned bicelles. J. Magn. Reson. 256 (2015) 1422.

[38] J. Raya, B. Perrone, and J. Hirschinger, Chemical shift powder spectra enhanced by multiple-contact cross-polarization under slow magic-angle spinning. J. Magn. Reson. 227 (2013) 93-102.

[39] K. Takegoshi, and C.A. McDowell, A 2D-exchange separated local field (EXSLF) experiment: An application to a 13C-1H isolated spin system in the solid state. J. Chem. Phys. 86 (1987) 6077.

[40] L. Müller, A. Kumar, T. Baumann, and R.R. Ernst, Transient Oscillations in NMR CrossPolarization Experiments in Solids. Phys. Rev. Lett. 32 (1974) 1402. 
[41] A. Naito, and C.A. McDowell, Anisotropic behavior of the $13 \mathrm{C}$ nuclear spin dynamics in a single crystal of l-alanine. J. Chem. Phys. 84 (1986) 4181.

[42] P.R. Levstein, G. Usaj, and H.M. Pastawski, Attenuation of polarization echoes in nuclear magnetic resonance: A study of the emergence of dynamical irreversibility in manybody quantum systems. J. Chem. Phys. 108 (1998) 2718.

[43] G.A. Alvarez, E.P. Danieli, P.R. Levstein, and H.M. Pastawski, Environmentally induced quantum dynamical phase transition in the spin swapping operation. J. Chem. Phys. 124 (2006) 194507.

[44] A.K. Chattah, G.A. Alvarez, P.R. Levstein, F.M. Cucchietti, H.M. Pastawski, J. Raya, and J. Hirschinger, Many-spin quantum dynamics during cross polarization in 8CB. J. Chem. Phys. 119 (2003) 7943.

[45] P. Reinheimer, J. Hirschinger, P. Gilard, and N. Goetz, Cross-polarization dynamics and proton dipolar local field measurements in some organic compounds. Magn. Reson. Chem. 35 (1997) 757.

[46] K. Blum, Density matrix theory and applications, Plenum Press, New York and London, 1981.

[47] A. Abragam, The Principles of Nuclear Magnetism, Oxford University Press, Oxford, 1961.

[48] R.R. Ernst, G. Bodenhausen, and A. Wokaun, Principles of Nuclear Magnetic Resonance in One and Two Dimensions, Oxford University Press, Oxford, 1987.

[49] U. Fano, Description of states in quantum mechanics by density matrix and operator techniques. Rev. Mod. Phys. 29 (1957) 74-93.

[50] F. Engelke, T. Kind, D. Michel, M. Pruski, and B.C. Gerstein, A theoretical approach for the calculation of the Hartmann-Hahn matching under cross polarization and magic-angle spinning. J. Magn. Reson. 95 (1991) 286-298.

[51] M.H. Levitt, D. Suter, and R.R. Ernst, Spin dynamics and thermodynamics in solid-state NMR cross polarization. J. Chem. Phys. 84 (1986) 4243.

[52] S. Hediger, B.H. Meier, N. Kurur, G. Bodenhausen, and R.R. Ernst, NMR cross polarization by adiabatic passage through the Hartmann-Hahn condition (APHH). Chem. Phys. Lett. 223 (1994) 283-288.

[53] M.M. Maricq, and J.S. Waugh, NMR in rotating solids. J. Chem. Phys. 70 (1979) 3300.

[54] F. Marica, and R.F. Snider, An analytical formulation of CPMAS. Solid State Nucl. Magn. Reson. 23 (2003) 28.

[55] J. Hirschinger, and J. Raya, Analytical descriptions of cross-polarisation dynamics: relaxing the secular approximations. Mol. Phys. 113 (2015) 3161-3175.

[56] P. Palmas, P. Tekely, and D. Canet, Local-Field Measurements on Powder Samples from Polarization Inversion of the Rare-Spin Magnetization. J. Magn. Reson. Ser. A 104 (1993) 26-36.

[57] P. Palmas, C. Malveau, P. Tekely, and D. Canet, Magnitudes and relative orientations of dipolar and shielding interaction tensors determined from the orientation dependence of spinning sidebands of slowly rotating powder samples. Solid State Nucl. Magn. Reson. 13 (1998) 45-53.

[58] C. Gardiennet, F. Marica, C.A. Fyfe, and P. Tekely, Determining the geometry of strongly hydrogen-bonded silanols in a layered hydrous silicate by solid-state nuclear magnetic resonance. J. Chem. Phys. 122 (2005) 054705.

[59] S. Zhang, B.H. Meier, and R.R. Ernst, Local monitoring of proton spin diffusion in static and rotating samples via spy detection. Solid State Nucl. Magn. Reson. 1 (1992) 313.

[60] M. Ernst, A. Verhoeven, and B.H. Meier, High-speed magic-angle spinning 13C NMR spectra of adamantane: self-decoupling of the heteronuclear scalar interaction and proton spin diffusion. J. Magn. Reson. 130 (1998) 176-185. 
[61] I. Schnell, Dipolar recoupling in fast-MAS solid-state NMR spectroscopy. Prog. Nucl. Magn. Reson. Spectrosc. 45 (2004) 145-207.

[62] T. Gullion, and J. Schaefer, Detection of weak heteronuclear dipolar coupling by rotational-echo double-resonance nuclear magnetic resonance. Adv. Magn. Reson. 13 (1989) 57-83.

[63] S. Zhang, X. Wu, and M. Mehring, Successive polarization under mismatched HartmannHahn condition. Chem. Phys. Lett. 166 (1990) 92.

[64] P. Bertani, J. Raya, P. Reinheimer, R. Gougeon, L. Delmotte, and J. Hirschinger, 19F/29Si distance determination in fluoride-containing octadecasil by Hartmann-Hahn crosspolarization under fast magic-angle spinning. Solid State Nucl. Magn. Reson. 13 (1999) 219-229.

[65] N.C. Nielsen, H. Bildsoe, H.J. Jakobsen, and M.H. Levitt, Double-quantum homonuclear rotary resonance: efficient dipolar recovery in magic-angle spinning nuclear magnetic resonance. J. Chem. Phys. 101 (1994) 1805-1812.

[66] M. Hologne, P. Bertani, T. Azaïs, C. Bonhomme, and J. Hirschinger, 1H/31P distance determination by solid state NMR in multiple-spin systems. Solid State Nucl. Magn. Reson. 28 (2005) 50-56.

[67] A. Hagemeyer, K. Schmidt-Rohr, and H.W. Spiess, Two-dimensional nuclear magnetic resonance experiments for studying molecular order and dynamics in static and rotating solids. Adv. Magn. Reson. 13 (1989) 85-130.

[68] R. Zwanzig, On the identity of three generalized master equations. Physica 30 (1964) 1109-1123.

[69] H. Mori, Transport, Collective motion, and Brownian motion. Prog. Theor. Phys. 33 (1965) 423-455.

[70] D.E. Demco, J. Tegenfeldt, and J.S. Waugh, Dynamics of cross relaxation in nuclear magnetic double resonance. Phys. Rev. B 11 (1975) 4133-4151.

[71] T.T.P. Cheung, and R. Yaris, Dynamics of cross relaxation in modulated systems: Application to nuclear magnetic double resonance of glassy polymers. J. Chem. Phys. 72 (1980) 3604.

[72] P.W. Anderson, and P.R. Weiss, Exchange narrowing in paramagnetic resonance. Rev. Mod. Phys. 25 (1953) 269-276.

[73] J. Hirschinger, A simple analytical model to describe dynamic magic-angle spinning dynamics. Concept in Magn. Reson. Part A 28A (2006) 307.

[74] J. Hirschinger, Analytical solutions to several magic-angle spinning NMR experiments. Solid State Nucl. Magn. Reson. 34 (2008) 210.

[75] M.F. Cobo, K. Malinakova, D. Reichert, K. Saalwächter, and E.R. DeAzevedo, Intermediate motions and dipolar couplings as studied by Lee-Goldburg cross-polarization NMR: Hartmann-Hahn matching profiles. Phys. Chem. Chem. Phys. 11 (2009) 7036.

[76] D. Suwelack, W.P. Rothwell, and J.S. Waugh, Slow molecular motion detected in the NMR spectra of rotating solids. J. Chem. Phys. 73 (1980) 2559-2569.

[77] M.F. Cobo, D. Reichert, K. Saalwächter, and E.R. deAzevedo, A double-component Anderson-Weiss approach for describing NMR signals of mobile SIn units: Application to constant-time DIPSHIFT experiments. J. Magn. Reson. 248 (2014) 115-125.

[78] J. Raya, and J. Hirschinger, Application of rotor-synchronized amplitude-modulated crosspolarization in a 13C-1H spin pair under fast magic-angle spinning. J. Magn. Reson. 133 (1998) 341.

[79] R. Gupta, G. Hou, T. Polenova, and A.J. Vega, RF inhomogeneity and how it controls CPMAS. Solid State Nucl. Magn. Reson. 72 (2015) 17-26.

[80] A. Bax, Two-Dimensional NMR in liquids, Delft University Press, Delft, 1984. 
[81] B.M. Fung, A.K. Khitrin, and K. Ermolaev, An improved broadband decoupling sequence for liquid crystal and solids. J. Magn. Reson. 142 (2000) 97-101.

[82] S. Hediger, B.H. Meier, and R.R. Ernst, Rotor-synchronized amplitude-modulated nuclear magnetic resonance spin-lock sequences for improved cross polarization under fast magic angle sample spinning. J. Chem. Phys. 102 (1995) 4000-4011.

[83] J. Hirschinger, and M. Hervé, Cross-polarization dynamics and spin diffusion in some aromatic compounds. Solid State Nucl. Magn. Reson. 3 (1994) 121-135.

[84] X. Wu, S. Zhang, and X. Wu, Two-stage feature of Hartmann-Hahn cross relaxation in magic-angle sample spinning. Phys. Rev. B 37 (1988) 9827-9829.

[85] P. Tekely, V. Gérardy, P. Palmas, D. Canet, and A. Retournard, Measurement of HartmannHahn cross-poalrization dynamics with quenching of proton T1r relaxation dependence. Solid State Nucl. Magn. Reson. 4 (1995) 361-367.

[86] F. Tian, and T.A. Cross, Dipolar oscillations in cross-polarized peptide samples in oriented lipid bilayers. J. Magn. Reson. 125 (1997) 220-223.

[87] B.B. Kharkov, V.I. Chizhik, and S.V. Dvinskikh, Broadband cross-polarization-based heteronuclear dipolar recoupling and dynamic NMR studies of rigid and soft solids. J. Chem. Phys. 144 (2016) 034201.

[88] P. Tekely, P. Palmas, and D. Canet, Effect of proton spin exchange on the residual 13C MAS NMR linewidths. Phase-modulated irradiation for efficient heteronuclear decoupling in rapidly rotating solids. J. Magn. Reson. Ser. A 107 (1994) 129-133.

[89] S. Clough, and K.W. Gray, The stochatic theory of the nuclear magnetic resonance line in rotating solids. Proc. Phys. Soc. 79 (1962) 457-467.

[90] A. Kubo, R. Ikeda, and D. Nakamura, 1H nuclear magnetic resonance studies on structural phase transitions and molecular dynamics of five-membered rings in solid ferrocene, azaferrocene and ruthenocene. J. Chem. Soc., Faraday Trans. 282 (1986) 1543-1562.

[91] P. Li, Q. Chen, and S. Zhang, Analytical solution of cross-polarization dynamics. J. Magn. Reson. 250 (2015) 76-79.

[92] C. Tripon, M. Aluas, X. Filip, and C. filip, Polarization transfer from remote protons in 13C CP/MAS. J. Magn. Reson. 183 (2006) 68-76.

[93] K. Akasaka, S. Ganapathy, C.A. McDowell, and A. Naito, Spin-spin and spin-lattice contributions to the rotating frame relaxation of ${ }^{13} \mathrm{C}$ in L-alanine. J. Chem. Phys. 78 (1983) 3567-3272.

[94] M.H. Levitt, Composite pulses. in: D.M. Grant, and R.K. Harris, (Eds.), Encyclopedia of Nuclear Magnetic Resonance, Wiley, 1996.

[95] S.N. Koroloff, D.M. Tesch, E.O. Awosanya, and A.A. Nevzorov, Sensitivity enhancement for membrane proteins reconstituted in parallel and perpendicular oriented bicelles obtained by using repetitive cross-polarization and membrane-incorporated free radicals. J. Biomol. NMR 67 (2017) 135-144. 